Robin S. Langley

Department of Engineering, University of Cambridge, CB2 1PZ rs121@eng.cam.ac.uk

The crossing rates, exceedance probabilities, and related statistical properties of the energy frequency response functions of a random built-up system

\begin{abstract}
A method is derived for computing a number of key statistical properties of the vibrational energy of each component of a builtup system that has random properties. The energy is considered to be a random function of frequency, and the derived statistical properties include: the mean rate at which the energy crosses a specified level, the probability that the energy will exceed a specified level within a given frequency band, the mean trough-topeak height, the rate of occurrence of peaks, and the mean quefrency (a measure of the rate of fluctuation of the energy). The analysis is based on combining Statistical Energy Analysis (SEA) with a non-parametric model of uncertainty based on the Gaussian Orthogonal Ensemble (GOE), and avoids the use of Monte Carlo simulations or large computational models. By way of example, the method is applied to a number of coupled plate systems.
\end{abstract}




\title{
The crossing rates, exceedance probabilities, and related statistical properties of the energy frequency response functions of a random built-up system
}

\begin{abstract}
A method is derived for computing a number of key statistical properties of the vibrational energy of each component of a builtup system that has random properties. The energy is considered to be a random function of frequency, and the derived statistical properties include: the mean rate at which the energy crosses a specified level, the probability that the energy will exceed a specified level within a given frequency band, the mean trough-topeak height, the rate of occurrence of peaks, and the mean quefrency (a measure of the rate of fluctuation of the energy). The analysis is based on combining Statistical Energy Analysis (SEA) with a non-parametric model of uncertainty based on the Gaussian Orthogonal Ensemble (GOE), and avoids the use of Monte Carlo simulations or large computational models. By way of example, the method is applied to a number of coupled plate systems.
\end{abstract}

\section{Introduction}

The prediction of the dynamic response of a complex system to external excitation is complicated by the fact that the properties of the system may not be known to a high degree of precision due to material or manufacturing uncertainties. This is a particular problem when high frequency excitation is of concern; the system response has a short spatial wavelength and this means that the response can be very sensitive to small changes in the system. This issue has been the subject of research for many years, and much early work was performed on the statistical properties of the response of "single" components such as rooms [1] and isolated structural components [2]. There is now a large literature on the analysis of random built-up systems and two main approaches have evolved. In the first approach a deterministic model of the system is randomized by assigning uncertainty to a number of random physical parameters, and the aim is to propagate the uncertainty through the model to compute the uncertainty in the response. This approach typically requires very considerable computational effort, and many 
sophisticated techniques have been developed to increase the efficiency of the calculations. This type of approach will not be considered in detailed here, but an introduction to a range of available analysis methodologies is given in reference [3], and the application of the principle of maximum entropy to uncertainty in this context is described in reference [4]. The second approach is adopt a more analytical standpoint based on non-parametric models of the uncertainty. If a system component is sufficiently random, then the mode shapes and natural frequencies are known to conform to a universal distribution which depends only on the modal density (the average number of modes in a unit frequency band) of the system [5]. The distribution arises from the Gaussian Orthogonal Ensemble (GOE) family of matrices, and there is much literature in the field of random matrices [6] that can be exploited for engineering purposes. It has been shown that the GOE properties can be used to yield closed form results for the mean and variance of the response of a random component [7,8], and as discussed below, the approach has been combined with Statistical Energy Analysis (SEA) to yield the mean and variance of the response of built-up systems [9].

A further difficulty with the analysis of the response of a complex system at high frequencies is that very many degrees of freedom are needed to capture the detailed behavior of the system. The SEA approach mentioned above was introduced by Lyon [10] to address this problem. Rather than seek the detailed response of the system, the method represents the system as a collection of coupled subsystems, and the aim is to compute the vibrational (or acoustic) energy of each subsystem. The method requires relatively few degrees of freedom, and the coefficients in the governing equations (which are based on power balance) can be found from wave or modal considerations [10]. The solution of the SEA equations yields the average of the energy over an ensemble of random structures; by employing GOE techniques the method was extended in reference [9] to predict the variance of the response. The results for the mean and variance yielded by the method are generally smooth functions of frequency, i.e. individual modal peaks do not appear, and frequency fluctuations in the underlying deterministic responses are smoothed by the averaging process. In reference [11] the method was extended for a single subsystem to yield more advanced statistical properties of the energy when viewed as a random function of frequency. The properties derived were: the mean rate at which the energy crosses a specified level, the probability that the energy will cross a specified level in a given frequency band, the mean trough-to-peak height, the mean rate of occurrence of peaks, and the mean quefrency of the energy. The term "quefrency" relates to the rate of fluctuation of the energy as a function of frequency [12] - it is the direct analogy of the frequency of a function of time. In what follows the analysis given in [11] is extended from a single subsystem to a general built-up system, thus allowing advanced statistical properties to be derived within the context of an SEA-type approach. In order to extend SEA in this way, it is first necessary to derive expressions for the variance of the first and second frequency derivatives of the subsystem energies, and this forms part of the present analysis. It should be emphasized that because the present approach is based on SEA, then the range of applicability is restricted to those systems for which SEA is a valid. There has been much discussion as to the conditions required for the successful application of SEA (for example [13-16]) and the main points are: (i) each subsystem must be reverberant and must be sufficiently random, (ii) the coupling between the subsystems must be sufficiently weak to avoid strong correlations between 
the subsystem responses. Further elaboration of these points is given in the following sections.

The present analysis has application to the design of systems that must meet noise and vibration targets. The exceedance probability allows the safety and performance of the system to be assessed. For example, a system may be subjected to a harmonic excitation of uncertain frequency; if the frequency is known to be restricted with a band, then the present method can be used to predict that probability that the system response will not exceed performance limits for any frequency within the band. Furthermore, the detailed information provided by the crossing rates and quefrency can be used in response reconstruction, i.e. the generation of a detailed sample of the response based on ensemble statistics, and this could be employed, for example, in auralization.

Given that the present analysis is a generalization of the method presented in reference [11], the main results of that reference are summarized in Section 2. The method is then extended from a single subsystem to a general built-up system in Section 3 , and a range of example applications is given in Section 4, consisting of two-plate and three-plate systems. Concluding comments are then given in Section 5.

\section{Summary of energy FRF statistics for a single subsystem}

If a proportionally damped linear system is subjected to harmonic forcing of frequency $\omega$, then the complex amplitude of the velocity at a spatial point $\mathbf{x}, u(\omega, \mathbf{x})$ say, can be written in the form [17]

$$
u(\omega, \mathbf{x})=\sum_{n} \frac{i \omega g_{n} \phi_{n}(\mathbf{x})}{\omega_{n}^{2}-\omega^{2}+i \eta \omega_{n}^{2}}, \quad g_{n}=\int_{R} P(\mathbf{x}) \phi_{n}(\mathbf{x}) \mathrm{d} \mathbf{x}
$$

where $\eta$ is the loss factor and $\omega_{n}$ and $\phi_{n}(\mathbf{x})$ are respectively the natural frequency and mode shape (scaled to unit generalised mass) associated with the $n$th mode of vibration. The term $g_{n}$ is the generalised force in the $n$th mode arising from the harmonic forcing, which has an intensity of complex amplitude $P(\mathbf{x})$, and the integration region $R$ represents the spatial domain (length, area, or volume) occupied by the system. For ease of notation the velocity and mode shapes are taken to be scalar quantities (representing, for example, the out-of-plane motion of a plate), although this simplification can readily by lifted without any significant change to the following equations. If the system has mass density $\rho(\mathbf{x})$ then the time-averaged kinetic energy can be written as

$$
T(\omega)=(1 / 4) \int_{R} \rho(\mathbf{x})|u(\omega, \mathbf{x})|^{2} \mathrm{~d} \mathbf{x}=\sum_{n} a_{n} H\left(\omega, \omega_{n}\right)
$$

where

$$
a_{n}=\left|g_{n}\right|^{2}, \quad H\left(\omega, \omega_{n}\right)=\frac{\left(\omega^{2} / 4\right)}{\left(\omega_{n}^{2}-\omega^{2}\right)^{2}+\left(\eta \omega_{n}^{2}\right)^{2}}
$$


If the system has random properties due to manufacturing uncertainties or other sources of variability, then in order to fully analyse the vibrational response it is necessary to consider an ensemble of systems, with each member of the ensemble having a different set of natural frequencies and mode shapes. If the statistical properties of the modal parameters are known then in principle all of the statistical properties of the kinetic energy of the system can be found from Eq. (3). As discussed in references [5-8], if the system is sufficiently random and has non-localised modes then, regardless of the source of the randomness, the statistics of the natural frequencies and mode shapes tend to conform to the eigenvalue and eigenvector statistics associated with the Gaussian Orthogonal Ensemble (GOE) of random matrices [6]. The term "sufficiently random" is normally understood to mean that the statistical variation in the natural frequencies is greater than the mean spacing between the natural frequencies, so that there is "mixing" of the mode shapes across the ensemble: a particular mode of one member of the ensemble has a significant projection onto a number of the modes of each of the other members of the ensemble [18]. The probability density functions associated with the GOE depend only on one parameter, the mean spacing of the natural frequencies, and this fact allows surprisingly general results to be obtained for the response of random systems $[5,7]$.

In reference [11] the statistics of the GOE were exploited to study the crossing rates of the kinetic energy function given by Eq. (3). It was shown that a good approximation to the mean rate at which the function up-crosses a level $T=b$ is given by

$$
v_{b}^{+}(\omega)=\sigma_{s}\left[2 \pi\left(\sigma^{2}+\mu^{2}\right)\right]^{-1 / 2} b p(b),
$$

where

$$
\begin{gathered}
p(b)=\frac{1}{\sqrt{2 \pi}\left(b c_{1}\right)} \exp \left\{-\frac{1}{2}\left(\frac{\ln b-c_{2}}{c_{1}}\right)^{2}\right\}, \\
c_{1}=\sqrt{\ln \left(1+\sigma^{2} / \mu^{2}\right)}, \quad c_{2}=\ln \left(\frac{\mu}{\sqrt{1+\sigma^{2} / \mu^{2}}}\right), \\
\mu=\mathrm{E}[T], \quad \sigma^{2}=\operatorname{Var}[T], \quad \sigma_{s}^{2}=\operatorname{Var}[\partial T / \partial \omega] .
\end{gathered}
$$

Here $\mu$ and $\sigma^{2}$ are the mean and variance of the kinetic energy, and $\sigma_{s}^{2}$ is the mean squared value of the frequency derivative of the energy (defined as $s=\partial T / \partial \omega$, with the mean value of $s$ being taken to be zero); the crossing rate as given by Eq. (6) is fully determined by the three parameters $\mu, \sigma^{2}$, and $\sigma_{s}^{2}$. Closed form expressions for $\mu$ and $\sigma^{2}$ have been derived in references [7,19] in the form

$$
\begin{gathered}
\mu=\frac{\mathrm{E}\left[a_{n}\right] \pi n(\omega)}{8 \eta \omega}, \\
\sigma^{2}=\mu^{2} r^{2}(\alpha, \gamma, m)=\mu^{2}\left\{\frac{[\alpha+(\gamma+1) q(m)]}{\pi m}+\gamma\right\},
\end{gathered}
$$


where $n(\omega)$ is the modal density of system (i.e. the average number of natural frequencies that fall in a unit frequency interval, or equivalently, the inverse of the mean frequency spacing), and the other parameters in the equations are given by

$$
\begin{gathered}
q(m)=-1+\frac{1}{2 \pi m}[1-\exp (-2 \pi m)]+\mathrm{E}_{1}(\pi m)\left[\cosh (\pi m)-\frac{1}{\pi m} \sinh (\pi m)\right], \\
m=\omega \eta n, \quad \alpha=\frac{\mathrm{E}\left[a_{n}^{2}\right]}{\mathrm{E}\left[a_{n}\right]^{2}}, \quad \gamma \equiv \frac{\operatorname{Cov}\left[a_{r}, a_{s}\right]}{\mathrm{E}\left[a_{r}\right] \mathrm{E}\left[a_{s}\right]} \quad(r \neq s) .
\end{gathered}
$$

The parameter $m$ is known as the modal overlap factor, and the parameters $\alpha$ and $\gamma$ that are defined by Eqs. (17) and (18) can be found by using the GOE statistical properties of the mode shapes in combination with Eqs. (2) and (4) [19]. To complete the set of three response quantities $\mu, \sigma^{2}$, and $\sigma_{s}^{2}$ needed to compute the mean crossing rate, it was shown in reference [11] that $\sigma_{s}^{2}$ is given by

$$
\sigma_{s}^{2}=\mu^{2} n^{2} \frac{\partial^{2}}{\partial m^{2}}\left[\frac{\alpha+(\gamma+1) q(m)}{\pi m}\right]
$$

A full derivation of the crossing rate formula, Eq. (6), is given in reference [11], which also includes a comprehensive list of the approximations and assumptions employed in the analysis. A brief list of the key assumptions is as follows:

(i) The natural frequencies and mode shapes of the system conform to GOE statistics.

(ii) The marginal probability density function of the kinetic energy $p(T)$ is lognormal, which is a consequence of GOE statistics providing the modal overlap of the system is not small, as shown in reference [20].

(iii) The conditional probability density function $p(s \mid T)$ has bounds that grow linearly with $T$, and the conditional variance of $s$ is proportional to $T$.

(iv) The marginal probability density function $p(s)$ is a zero mean Gaussian distribution, based on the empirical evidence presented in [11].

Whereas assumptions (i) and (ii) are based on the occurrence of GOE statistics, assumption (iii) is based on a study of bounds and assumption (iv) is based on empirical evidence (although it can be noted that the final result is not sensitive to the precise distribution of $s$ ).

If the modal overlap of the system is greater than unity, then Eqs. (14) and (19) can be well approximated by the simpler results 


$$
\sigma^{2} \approx \mu^{2}\left\{\frac{(\alpha-\gamma-1)}{\pi m}+\gamma\right\}, \quad \sigma_{s}^{2} \approx\left(\frac{2}{\omega^{2} \eta^{2}}\right) \sigma^{2}
$$

Furthermore, if $m>2$ then a good approximation to the maximum crossing rate (i.e. the rate of crossing the most crossed level $b$ ) can be written as

$$
\left(v_{b}^{+}\right)_{\max }=\beta \sigma_{s}\left[2 \pi \mu^{2}\left(1+\sigma^{2} / \mu^{2}\right) \ln \left(1+\sigma^{2} / \mu^{2}\right)\right]^{-1 / 2} \approx \frac{1}{2 \pi}\left(\frac{\sqrt{2}}{\omega \eta}\right) .
$$

Were $\left(v_{b}^{+}\right)_{\max }$ to have units of Hz then Eq. (22) would identify the quantity $\sqrt{2} /(\omega \eta)$ as the mean "circular frequency" of the fluctuations in the energy FRF. However, the FRF is expressed as a function of $\omega$, meaning that $\left(v_{b}^{+}\right)_{\max }$ has units of $(\mathrm{rad} / \mathrm{s})^{-1}$ and hence $\sqrt{2} /(\omega \eta)$ obviously has units of s/rad. Rather than being a circular frequency, $\sqrt{2} /(\omega \eta)$ represents a "quefrency" as used in cepstral analysis [12]; nonetheless it can be physically interpreted as the mean rate of fluctuation of the FRF in terms of phase rotation, and as discussed in reference [11] this result is consistent with earlier work in the field of random FRFs [1].

Equation (6) can be used to compute the mean number of times a response level $b$ is up-crossed in a frequency interval $\omega_{A}<\omega<\omega_{B}$, to give

$$
N^{+}(b)=\int_{\omega_{A}}^{\omega_{B}} v_{b}^{+}(\omega) \mathrm{d} \omega
$$

Additionally, if the up-crossings of the level $b$ are taken to represent a Poisson process then the probability that the response will lie below $b$ throughout the whole interval is given by [11]

$$
P=P_{0}(b) \exp \left\{-\int_{\omega_{A}}^{\omega_{B}} v_{b}^{+}(\omega) \mathrm{d} \omega\right\}
$$

where $P_{0}(b)$ is the probability that the response lies below $b$ at $\omega=\omega_{A}$. If Eq. (24) is employed with $P_{0}(b)=1$ then the equation has the alternative interpretation that $P$ is the probability of neither up-crossing nor down-crossing the level $b$, regardless of the initial conditions at $\omega=\omega_{A}$. The approximations involved in Eq. (24) are discussed in reference [11] and will be considered further in Section 4.1 of the present work.

In addition to level crossing rates, reference [11] considered a number of the statistical properties of the peaks of the energy FRF. It was shown that the mean rate of occurrence of peaks can be approximated by 


$$
v_{P}=\frac{1}{2 \pi}\left(\frac{\sigma_{r}}{\sigma_{s}}\right)
$$

where $\sigma_{r}$ is the standard deviation of the second derivative of the FRF, which was shown to be given by

$$
\sigma_{r}^{2} \equiv \operatorname{Var}\left[\partial^{2} T / \partial \omega^{2}\right]=\mu^{2} n^{4} \frac{\partial^{4}}{\partial m^{4}}\left[\frac{\alpha+(\gamma+1) q(m)}{\pi m}\right]
$$

Finally it was shown that the mean trough-to-peak height can be approximated by

$$
\mathrm{E}[R]=\sqrt{\pi / 6}\left(\omega \eta \sigma_{\mathrm{s}}\right)
$$

All of the equations in this section relate to the statistics of a system with proportional damping and non-localised modes, which means that the analysis is typically applicable to a single component such as a plate, shell, or acoustic cavity. Most practical structures are built-up from many components and consequently have non-proportional damping and localised modes, and the question then arises as to whether the above equations can be extended to this case. It is shown in the following section that this can be achieved by combining the equations with Statistical Energy Analysis (SEA).

\section{Energy FRF statistics in built-up systems}

A built-up system with randomly uncertain properties can often be represented as an assembly of relatively simple components or "subsystems", and there is engineering interest in predicting the ensemble statistics of the vibrational energy of each subsystem when an excitation is applied to one or more of the subsystems. This topic has been the subject of research for more than half a century, and Statistical Energy Analysis (SEA) is now an established technique for predicting the ensemble average values of the subsystem energies [10]. As mentioned in the Introduction, the conditions required for the validity of these equations include subsystem reverberance and randomness, and weak coupling between the subsystems [13-16]. The SEA equations express a vibrational power balance for each subsystem, and when written in matrix notation the equations have the form

$$
\mathbf{C} \hat{\mathbf{E}}=\mathbf{P}_{\text {in }},
$$

Where

$$
\begin{gathered}
\hat{E}_{j}=2 \mathrm{E}\left[T_{j}\right] / n_{j}, \\
C_{j j}=\omega \eta_{j} n_{j}+\sum_{k \neq j} \omega \eta_{j k} n_{j},
\end{gathered}
$$




$$
C_{j k}=-\omega \eta_{j k} n_{j}, \quad j \neq k
$$

Here $\mathrm{E}\left[T_{j}\right]$ is the ensemble average of the kinetic energy of subsystem $j$, and $n_{j}$ and $\eta_{j}$ are respectively the modal density and the loss factor of the subsystem. The modal density can be found analytically for most types of component [10], and so this quantity can be assumed to be known in Eq. (28). The coefficients $\eta_{j k}$ are referred to as coupling loss factors, and there are many ways of calculating these values, ranging from modal methods [10], wave transmission approaches [21], and a method based on a diffuse field reciprocity relation [22], and so again these quantities can be assumed to be known. The right hand side of Eq. (28) is a vector of ensemble averaged power inputs arising from external excitation, and for loading of the type considered in Section 2 the power input to subsystem $j$ can be written as [10]

$$
P_{\mathrm{in}, j}=\mathrm{E}\left[a_{n, j}\right] \pi n_{j} / 4
$$

where $a_{n, j}$ relates to the generalized force applied to the subsystem, as in Eq. (4). If the SEA equations are applied to a system consisting of only one subsystem, then it can readily be verified that Eq. (13) is recovered for the ensemble average of the kinetic energy.

Although Eq. (28) can be used to find the ensemble average of the energy in each subsystem, this information is not sufficient to predict the energy crossing rates: the variance of the energy and the variance of the frequency derivative of the energy are also needed. SEA was extended in reference [9] to yield the variance of the subsystem energies, and the method will be further extended in what follows to yield the variance of the first and second derivatives of the subsystem energies (the second derivative being needed to predict the statistics of the peaks). The approach taken in reference [9] was to recognise that Eq. (28) governs the ensemble average energy of the system and hence it cannot be applied independently to each member of the ensemble; however a generalized (non-SEA) energy-power relation can be established for each member of the ensemble, at least in principle [9], providing there is no correlation in the loading applied to different subsystems. Surprisingly, the fact that such a relation exists is sufficient to produce an SEA variance theory without any need to explicitly find the relation for each ensemble member. The energy-power relation for any particular ensemble member is written in the form

$$
\mathbf{D E}=\mathbf{P},
$$

where $E_{j}=2 T_{j} / n_{j}$ is the vibrational energy of subsystem $j$, and the matrix $\mathbf{D}$ and the vector $\mathbf{P}$ will vary randomly from ensemble member to ensemble member. Each term in Eq. (33) can be considered to fluctuate across the ensemble around the values that appear in Eq. (28), so that

$$
\mathbf{E}=\hat{\mathbf{E}}+\mathbf{E}_{\text {ran }}
$$




$$
\begin{aligned}
& \mathbf{P}=\mathbf{P}_{\text {in }}+\mathbf{P}_{\text {ran }}, \\
& \mathbf{D}=\mathbf{C}+\mathbf{D}_{\text {ran }} .
\end{aligned}
$$

The detailed analysis contained in reference [9] reveals a surprising subtlety: although the matrix $\mathbf{C}$ is symmetric, the matrix $\mathbf{D}_{\text {ran }}$ is in general non-symmetric. In addition, energy conservation considerations imply that the entries of $\mathbf{D}_{\text {ran }}$ are not fully independent, but rather the diagonal entries can be expressed in terms of the off-diagonal entries in the form

$$
D_{\text {ran }, k k}=-\sum_{j \neq k} D_{\text {ran }, j k}
$$

It is shown in reference [9] that the variance of the power inputs and the off-diagonal matrix entries can be written as

$$
\begin{gathered}
\operatorname{Var}\left(P_{\mathrm{ran}, j}\right)=P_{\mathrm{in}, j}^{2} r^{2}\left(\alpha_{j}, \gamma_{j}, m_{j}\right), \\
\operatorname{Var}\left(D_{\mathrm{ran}, j k}\right)=C_{j k}^{2} r^{2}\left(\alpha_{j k}, \gamma_{j k}, m_{j}\right),
\end{gathered}
$$

where the function $r^{2}(\alpha, \gamma, m)$ is given in Eq. (14), and the term $m_{j}$ is an effective insitu modal overlap factor for subsystem $j$, which is given by [9]

$$
m_{j}=1 / \mathbf{C}_{j j}^{-1}
$$

For an uncoupled subsystem $m_{j}$ is simply the standard modal overlap factor; when the subsystem is coupled to other subsystems then the coupling leads to an increase in the effective loss factor, and this effect is captured by Eq. (40). The various $\alpha$ and $\gamma$ terms that appear in Eqs. (38) and (39) depend on the nature of the subsystem coupling and the excitation, and these terms will be discussed further in Section 4.1 and in the Appendix. The asymmetry in the statistics of the matrix $\mathbf{D}$ is clearly visible in Eq. (39), which states that the variance of the entry $D_{\text {ran }, j k}$ depends on the modal overlap factor of the receiving subsystem $j$ but not on the modal overlap factor of the source subsystem $k$. The physics behind this result is explained in reference [9]; in brief, the coefficient governs the energy flow into subsystem $j$ when the energy in subsystem $k$ is prescribed (and therefore nonrandom).

Equations (33)-(40) enable expressions for the variance of the subsystem energies to be derived. Initially Eqs. (34)-(36) can be substituted into Eq. (33), and by making use of Eq. (28) and neglecting second order random terms this yields

$$
\mathbf{E}_{\mathrm{ran}}=\mathbf{C}^{-1} \mathbf{P}_{\mathrm{ran}}-\mathbf{C}^{-1} \mathbf{D}_{\mathrm{ran}} \hat{\mathbf{E}}
$$


Now by using Eq. (37) to express the diagonal entries of $\mathbf{D}_{\text {ran }}$ in terms of the off-diagonal entries, the $j$ th row of Eq. (41) can be written in the form

$$
E_{\mathrm{ran}, j}=\sum_{m} C_{j m}^{-1} P_{\mathrm{ran}, m}-\sum_{m} \sum_{n \neq m}\left(C_{j m}^{-1}-C_{j n}^{-1}\right) \hat{E}_{n} D_{\mathrm{ran}, m n} .
$$

The final result for the covariance of the subsystem energies can be obtained by taking the product of the $j$ th and $k$ th rows and assuming that the random power inputs are uncorrelated from the random matrix entries [9] to yield

$$
\operatorname{Cov}\left(E_{j} E_{k}\right)=\sum_{m} C_{j m}^{-1} C_{k m}^{-1} \operatorname{Var}\left(P_{\mathrm{ran}, m}\right)+\sum_{m} \sum_{n \neq m}\left(C_{j m}^{-1}-C_{j n}^{-1}\right)\left(C_{k m}^{-1}-C_{k n}^{-1}\right) \hat{E}_{n}^{2} \operatorname{Var}\left(D_{\mathrm{ran}, m n}\right) .
$$

This result represents a slight generalisation of that presented in reference [9], where only the variances of the energies were considered, rather than the covariances. Equation (43) yields the values of $\sigma_{j}$ that are required for the crossing rate equations - a result for the standard deviation of the frequency derivative of the energy of each subsystem is also required, and this is obtained in what follows.

If Eq. (28) and Eqs. (33)-(36) are all differentiated with respect to frequency then by combining the resulting equations and neglecting products of random terms it can readily be shown that

$$
\mathbf{E}_{\mathrm{ran}}^{\prime}=\mathbf{C}^{-1} \mathbf{P}_{\mathrm{ran}}^{\prime}-\mathbf{C}^{-1} \mathbf{D}_{\mathrm{ran}}^{\prime} \hat{\mathbf{E}}-\mathbf{C}^{-1} \mathbf{D}_{\mathrm{ran}} \hat{\mathbf{E}}^{\prime}-\mathbf{C}^{-1} \mathbf{C}^{\prime} \hat{\mathbf{E}}
$$

where a dash represents the frequency derivative of the variable. Following similar steps to those leading to Eq. (43) then yields

$$
\begin{aligned}
\operatorname{Var}\left(E_{j}^{\prime}\right) & =\sum_{m}\left(C_{j m}^{-1}\right)^{2} \operatorname{Var}\left(P_{\mathrm{ran}, m}^{\prime}\right)+\sum_{m} \sum_{n \neq m}\left(C_{j m}^{-1}-C_{j n}^{-1}\right)^{2} \hat{E}_{n}^{2} \operatorname{Var}\left(D_{\mathrm{ran}, m n}^{\prime}\right)+\varepsilon_{j}, \\
\varepsilon_{j} & =\sum_{m} \sum_{n \neq m}\left(C_{j m}^{-1}-C_{j n}^{-1}\right)^{2} \hat{E}_{n}^{\prime 2} \operatorname{Var}\left(D_{\mathrm{ran}, m n}\right)+\left(\mathbf{C}^{-1} \mathbf{C}^{\prime} \mathbf{S C}^{\left.\mathbf{T}^{\mathrm{T}} \mathbf{C}^{-1 \mathrm{~T}}\right)_{j j},}\right.
\end{aligned}
$$

where $\mathbf{S}$ is the covariance matrix of the subsystem energies, whose entries are given by Eq. (43). The appearance of $\mathbf{S}$ in Eq. (46) is the only reason why covariances were derived in Eq. (43), rather than just variances. The first two terms on the right hand side of Eq. (45) are directly analogous to the two contributions to Eq. (43), while the additional terms contained in $\varepsilon_{j}$ arise from the frequency derivative of ensemble average quantities. The contribution of $\varepsilon_{j}$ to Eq. (45) can generally be expected to be small. Based on Eq. (19), the power variance terms that appear in Eq. (45) can be written in the form

$$
\operatorname{Var}\left(P_{\mathrm{ran}, j}^{\prime}\right)=P_{\mathrm{in}, j}^{2} n_{j}^{2} \frac{\partial^{2}}{\partial m_{j}^{2}}\left[r^{2}\left(\alpha_{j}, \gamma_{j}, m_{j}\right)\right] .
$$


As shown in the Appendix, the corresponding result for the variance of the matrix entries is slightly more complicated and has the form

$$
\operatorname{Var}\left(D_{\mathrm{ran}, j k}^{\prime}\right)=C_{j k}^{2} n_{j}^{2} \frac{\partial^{2}}{\partial m_{j}^{2}}\left[r^{2}\left(\alpha_{j k}, \gamma_{j k}, m_{j}\right)\right]+2 C_{j k}^{2}\left\{\frac{N_{k}+2+q\left(m_{j}\right)}{N_{k} \omega^{2} \eta_{k}^{2} \pi m_{j}}+\left(\frac{1}{N_{k} \omega^{2} \eta_{k}^{2}}\right)\right\}
$$

Although the theory presented in the Appendix is general, the result shown in Eq. (48) is specifically for the case of two subsystems that are coupled such that the coupling involves $N_{k}$ degrees of freedom in subsystem $k$, and in this case $\alpha_{j k}=2\left(1+2 / N_{k}\right)$ and $\gamma_{j k}=1 / N_{k}$ (this issue is discussed further in Section 4.1). The position is now that the mean subsystem energies can be found from Eq. (28), the variance of the energies can be found from Eq. (43), and the variance of the slope of the energies can be found from Eq. (45). This provides all of the information needed for the crossing rate formula, Eq. (6), to be applied to each subsystem.

In order to evaluate the mean rate of peaks occurring in the subsystem energy FRFs, the variance of the second derivative of the energy of each subsystem is required. This can be found by following a similar approach to that described above for the slope of the energy; if terms involving the derivatives of ensemble averaged quantities are neglected, then the result obtained is directly analogous to Eq. (43) and has the form

$$
\operatorname{Var}\left(E_{j}^{\prime \prime}\right)=\sum_{m}\left(C_{j m}^{-1}\right)^{2} \operatorname{Var}\left(P_{\mathrm{ran}, m}^{\prime \prime}\right)+\sum_{m} \sum_{n \neq m}\left(C_{j m}^{-1}-C_{j n}^{-1}\right)^{2} \hat{E}_{n}^{2} \operatorname{Var}\left(D_{\mathrm{ran}, m n}^{\prime \prime}\right),
$$

where a double-dash indicates the second derivative with respect to frequency. The power variance terms that appear on the right hand side of this equation can be found by analogy with Eq. (26) to be given by

$$
\operatorname{Var}\left(P_{\mathrm{ran}, j}^{\prime \prime}\right)=P_{\mathrm{in}, j}^{2} n_{j}^{4} \frac{\partial^{4}}{\partial m_{j}^{4}}\left[r^{2}\left(\alpha_{j}, \gamma_{j}, m_{j}\right)\right]
$$

and it is shown in the Appendix that the variance of the matrix entries (written specifically for $N_{k}$ coupling freedoms in subsystem $k$ ) has the form

$$
\begin{aligned}
\operatorname{Var}\left(D_{\mathrm{ran}, j k}^{\prime \prime}\right)= & C_{j k}^{2} n_{j}^{4} \frac{\partial^{4}}{\partial m_{j}^{4}}\left[r^{2}\left(\alpha_{j k}, \gamma_{j k}, m_{j}\right)\right]+24 C_{j k}^{2}\left\{\frac{N_{k}+2+q\left(m_{j}\right)}{N_{k} \omega^{4} \eta_{k}^{4} \pi m_{j}}+\left(\frac{1}{N_{k} \omega^{4} \eta_{k}^{4}}\right)\right\} \\
& +8 C_{j k}^{2} n_{j}^{2} \frac{\partial^{2}}{\partial m_{j}{ }^{2}}\left[\frac{N_{k}+2+q\left(m_{j}\right)}{N_{k} \omega^{2} \eta_{k}^{2} \pi m_{j}}\right] .
\end{aligned}
$$

Finally it can be noted that the energy quantities employed in SEA are energies per mode, so the quantities required for the analysis of the subsystem FRF statistics can be expressed in the form 


$$
\mu_{j}=n_{j} \hat{E}_{j}, \quad \sigma_{j}^{2}=n_{j}^{2} \operatorname{Var}\left(E_{j}\right), \quad \sigma_{s, j}^{2}=n_{j}^{2} \operatorname{Var}\left(E_{j}^{\prime}\right), \quad \sigma_{r, j}^{2}=n_{j}^{2} \operatorname{Var}\left(E_{j}^{\prime \prime}\right) .
$$

These results can be employed directly in the equations presented in Section 2, thus allowing the analysis contained in that section to be applied to each subsystem of a builtup system, and not just to a single subsystem as considered in reference [11].

\section{Numerical examples}

\subsection{A two plate system}

In order to validate the foregoing theory, an example system consisting of two plates that are coupled by a number of linear springs is considered. Both plates are rectangular and simply-supported with planform dimensions $0.8 \mathrm{~m} \times 0.67 \mathrm{~m}$. One plate is arranged vertically above the other, and five springs of stiffness $k$ are attached between the two plates. Plate 1 has thickness $h=1 \mathrm{~mm}$ and Plate 2 has thickness $1.5 \mathrm{~mm}$, and both plates are made of steel, with Young's modulus $E=2 \times 10^{11} \mathrm{~N} / \mathrm{m}^{2}$, density $\rho=7800 \mathrm{~kg} / \mathrm{m}^{3}$, and Poisson's ratio $v=0.3$. The modal density of each plate can be calculated from the formula [10]

$$
n=\left(\frac{A}{4 \pi}\right)\left[\frac{12 \rho\left(1-v^{2}\right)}{E h^{2}}\right]^{1 / 2},
$$

where $A$ is the plate area; this yields $n=0.0278 \mathrm{modes} / \mathrm{rad} / \mathrm{s}$ for Plate 1 and $n=0.0186$ modes/rad/sec for Plate 2. In what follows the forced response over the frequency range 1500 to $2500 \mathrm{rad} / \mathrm{s}$ is considered, which covers around 20 to 30 resonant modes in each plate. The damping in the plates is assigned so that Plate 1 has a constant modal overlap factor of 2.5, and Plate 2 has a constant modal overlap factor of 4.0 (in practical terms this might result from the application of a damping treatment). Accurate benchmark results for the response of the system have been calculated by using the LagrangeRayleigh-Ritz method, with the modes of the uncoupled simply-supported plates used as basis functions. In order to randomize the system 10 masses have been added to each plate in random locations, with each mass having $2 \%$ of the mass of the relevant plate; previous studies have shown that this degree of randomization is sufficient to promote the occurrence of GOE modal statistics over the frequency range of interest [9]. In this way the statistics of the response have been computed using a Monte Carlo, with each sample having the location of the masses selected from a uniform distribution over the surface of the plates. The fact that the masses randomize the system mode shapes implies that the computed statistics are insensitive to the location of the coupling springs, providing the springs are not placed too close to each other or to the edge of the plates. For the computed results the springs have been placed at least $10 \%$ of a side-length away from each edge and from each other, meaning that the precise location has no influence on the statistical results. A Monte Carlo ensemble of up to 1000 realizations has been used generate the benchmark results. 
In order to apply the foregoing theory it is necessary to derive an SEA model of the system, and this requires the evaluation of the coupling loss factor between the plates. If the vibrational energy flow through the five springs is taken to be incoherent, then the coupling loss factor can be found by simply multiplying the result for a single spring by five. A standard result is available for a single point coupling [10] and the application of this result yields

$$
\omega \eta_{12} n_{1}=\left(\frac{2}{\pi}\right) \frac{5 k^{2} \operatorname{Im}\left(D_{1 \infty}\right) \operatorname{Im}\left(D_{2 \infty}\right)}{\mid D_{1 \infty} D_{2 \infty}+k\left(D_{1 \infty}+D_{2 \infty}\right)^{2}},
$$

where $D_{j \infty}$ is the point-wise dynamic stiffness of an infinite plate having the same properties as Plate $j$. Again, a standard result is available for this quantity [10], so that

$$
D_{j \infty}=2 i \omega \rho_{j} h_{j} A_{j} /\left(\pi n_{j}\right)
$$

The spring stiffness is assigned the value $k=1 \times 10^{4}(\omega / 1500) \mathrm{N} / \mathrm{m}$, which yields a coupling factor $\omega \eta_{12} n_{1}$ that is approximately constant over the frequency range of interest. A spring stiffness of this type is clearly an academic construct that has been chosen to yield response statistics that are independent of frequency. There is no reason for this restriction other than the fact that the influence of a number of parameters on the system response is to be explored in what follows, and having a statistical response that is independent of frequency greatly simplifies the presentation of the results. The general theory presented in the previous section allows for the response to be frequency dependent, and because the calculation is a frequency-by-frequency procedure then the present validations also validate the more general case.

The foregoing theory also requires values for the various parameters $\alpha$ and $\gamma$ that appear in Eqs. (38) and (39). The terms $\alpha_{j}$ and $\gamma_{j}$ in Eq. (38) relate to the statistics of the generalized forces that are applied directly to subsystem $j$, and formulae for these parameters are given in Eqs. (17) and (18) as a function of the generalized force parameter $a_{n}$, which is defined by Eqs. (2) and (4). In what follows, point force excitation of the plates will be considered, in which case the parameter $a_{n}$ is simply the square of the mode shape $\phi_{n}$ at the point of excitation. If the excited subsystem has GOE statistics then the mode shapes are Gaussian and uncorrelated [6] and it follow from Eqs. (17) and (18) that $\alpha_{j}=3$ and $\gamma_{j}=0$. However, it has been shown by Brody et. al. [23] that full GOE modal statistics are achieved only in the ideal case in which there is very strong mixing of the random modes across the ensemble, i.e. a mode of a particular member of the ensemble has a significant projection onto a very large number of the modes of any other member of the ensemble. Previous work [11] has shown that the degree of mixing for random plates of the present type is not ideal, leading typically to the values $\alpha_{j}=2.85$ and $\gamma_{j}=-0.05$, which will be adopted here (derived from the formulae presented in Section 7 of reference [23], corresponding to around 40 interacting 
modes). The terms $\alpha_{j k}$ and $\gamma_{j k}$ that appear in Eq. (39) relate to the statistics of the coupling loss factors, and it is shown in reference [9] that for point coupling $\alpha_{j k}=\left(2+4 / N_{k}\right)$ and $\gamma_{j k}=1 / N_{k}$ where $N_{k}$ is the effective number of active couplings. This is placed equal to the lesser of: (i) the number of physical point couplings, and (ii) the number of active modes in the source subsystem, which is given by the modal overlap factor $m_{k}$.

Results have been obtained for the case in which each plate is excited by a point force: the force acting on Plate 1 has amplitude $1-\alpha$ and that on Plate 2 has amplitude $\alpha$, and the range $0 \leq \alpha \leq 1$ has been explored, so that at the extreme values of the parameter only one plate is excited. The ensemble average of the energy in each plate is shown as a function of $\alpha$ in Figure 1 [insert Figure 1]. The fact that the modal overlap factors are held constant with frequency, together with the fact that the coupling factor given by Eq. (57) is also constant with frequency, implies that the statistical moments of the response are independent of frequency, and so the results shown in Figure 1 (and the following figures) apply to any frequency across the frequency band of interest. The analytical results shown in Figure 1 have been obtained using the SEA equation, Eq. (28), while the benchmark results have been obtained using the Lagrange-Rayleigh-Ritz approach. Since the aim of the present work is to focus on the validity of the crossingrate equations, and the accuracy of these equations depends on the accuracy of the underlying SEA predictions, the coupling loss factor predicted by Eq. (57) has been increased empirically by $5 \%$ to yield the level of agreement shown in Figure 1. Normally an error in an SEA prediction of $5 \%(0.2 \mathrm{~dB})$ would be considered perfectly acceptable, but the present concern is with the accuracy of the crossing rate predictions given an accurate SEA model. The relative variance of the plate energies, as predicted by Eq. (43), is shown in Figure 2 [insert Figure 2]. The energy variance equations have been validated previously [24] and the level of agreement shown in Figure 2 is generally good. For concentrated loading the relative variance is known to grow with increasing distance from the excited subsystem and this trend is evidenced in Figure 2 for the two cases $\alpha=0$ and $\alpha=1$, where the non-excited plate has the higher relative variance. The largest discrepancy in Figure 2 occurs for Plate 1 when $\alpha=1$, in which case the relative variance is around $20 \%$ in error, giving a $10 \%$ error in the relative standard deviation; more generally the agreement is much more accurate than this value. The variance of the slope of the plate energies, as predicted by Eq. (45) is shown in Figure 3 and there is good agreement with the benchmark results [insert Figure 3].

The effective quefrency of the two plates, as predicted from Eqs. (20)-(22) in the form $\sigma_{s} / \sigma$, is shown in Figure 4 [insert Figure 4]. A key feature of this figure is the effect of coupling on the quefrency of the plates. The plates are relatively weakly coupled, so that when the excitation is applied to Plate 1 alone $(\alpha=0)$ the quefrency of the plate has a value very close to the uncoupled result; the same remark applies to plate 2 , and in this case the uncoupled result for the plate is approached for $\alpha=1$. It can be noted that Plate 1 has a lower modal overlap factor than Plate 2 (2.5 versus 4.0), and hence the quefrency, which measures the rate of oscillation of the frequency response function, is higher for the first plate than the second plate in the absence of coupling. 
However in the presence of coupling, when Plate 1 is excited $(\alpha=0)$ the quefrency of Plate 2 is much greater than the uncoupled value. Likewise, when Plate 2 is excited the quefrency of Plate 1 is lower than the uncoupled value. These effects are demonstrated in Figures 5 and 6 [insert Figure 5 ] [insert Figure 6] which each show three benchmark realizations of the response of the system for the two cases $\alpha=0$ and $\alpha=1$; it is very noticeable that the response of Plate 2 is much more oscillatory in Figure 5 than in Figure 6. Another metric of the response that is yielded by the present analysis is the mean trough-to-peak height, and the value for each plate, as calculated from Eq. (27), is shown in Figure 7 as a function of $\alpha$ [inset Figure 7]. The level of agreement shown with the benchmark results is considered to be good; the present analysis is based on a range of approximations and assumptions, but has the advantage of requiring a negligible amount of computation time compared to a direct analysis.

The mean rate at which the energy crosses a specified level is given by Eq. (6), and the average number of crossings that will occur over a specified frequency range is given by the integral of the crossing rate over this range, Eq. (23). Results for the number of crossings vs the crossing level are shown in Figures 8 and 9 for the case in which excitation is applied to Plate 1 only $(\alpha=0)$ and the frequency range of interest is 1500 to $2500 \mathrm{rad} / \mathrm{s}$ [insert Figure 8] [inset Figure 9]. It can be seen that the present theory is in very good agreement with the benchmark results. The crossing rate can be employed in Eq. (24) to predict the probability that the energy will cross a specified level at least once (i.e. 1-P), and the results obtained are shown for the two plates in Figures 10 and 11 [insert Figure 10] [insert Figure 11]. In each case three sets of results are shown: (i) the results yielded by the present method, (ii) the results obtained by employing the benchmark results for the crossing rate in Eq. (24), and (iii) benchmark results for the probability of crossing. The very good level of agreement shown between (i) and (ii) in the figures reflects the level of agreement obtained for the crossing rates. The level of disagreement shown between (ii) and (iii) reflects the inadequacy of Eq. (24) over certain regions. The equation is based on the assumption that successive crossings of a specified level constitute a Poisson process; from random vibration theory it is known that this condition tends to be met at high levels, and this is demonstrated by the agreement between (ii) and (iii) for large energies. In random vibration applications Eq. (24) tends to overestimate the probability of a crossing, due to a phenomena known as "clumping" of the crossings [25]. The converse is seen in Figures 10 and 11, where Eq. (24) underestimates the probability of crossing. It is argued in reference [11] that this is because veering between the system natural frequencies causes an "anti-clumping" effect. Despite these effects the present approach yields a good guideline as to whether a specified energy level will be exceeded.

To explore the robustness of the present method against coupling strength and "non-tuned" coupling loss factors, three cases of increasing coupling strength have been considered with no adjustments made to the coupling loss factors yielded by the standard SEA theory. The three spring stiffness considered are $k=10^{3}(\omega / 1500) \mathrm{N} / \mathrm{m}$, $k=10^{4.5}(\omega / 1500) \mathrm{N} / \mathrm{m}$, and $k=10^{6}(\omega / 1500) \mathrm{N} / \mathrm{m}$, and results for the crossing rates of the energies of the two plates are shown in Figures 12 and 13 [insert Figure 12][insert Figure 13]. It can be seen from Figure 12 that the response of Plate 1 is insensitive to 
the value of the stiffness when the stiffness has either of the two lower values. This indicates that the system is weakly coupled, and this can be confirmed from Figure 13, where it can be seen that the energy levels are much lower for Plate 2 than for Plate 1 for these two cases. The highest level of stiffness represents strong coupling, since the response of Plate 1 is strongly affected by the presence of Plate 2 (as can be seen in Figure 12), and the response on Plate 2 is within $10 \mathrm{~dB}$ of the response of Plate 1 (Figure 13). In all cases the present theory captures the physical trends in the response and provides good qualitative agreement with the benchmark simulations, providing further evidence of the validity of the underlying assumptions.

\subsection{A three plate system}

In this section the previous example is extended by the addition of a third plate; this plate is coupled to Plate 2 via five springs having the properties previously described, and the plate has the same properties as Plates 1 and 2 other than the fact that the thickness of the plate is $1.2 \mathrm{~mm}$ (so that the three plates have thicknesses $1 \mathrm{~mm}, 1.5 \mathrm{~mm}$, and $1.2 \mathrm{~mm}$ ). All three plates are randomized by the addition of point masses, as described previously. The modal overlap factor for Plate 3 is set to 2.0, and the coupling loss factor between plates 2 and 3 is increased empirically by $5 \%$ over the value predicted by Eq. (57) for reasons described previously. In this case a force of unit amplitude is applied to Plate 3, and results for the number of crossings of a specified level are shown sequentially for the three plates in Figures 14 to 16, and the corresponding crossing probabilities are shown in Figures 17 to 19 [insert Figure 14] [insert Figure 15] [insert Figure 16] [insert Figure 17] [insert Figure 18] [insert Figure 19]. The decrease in energy away from the excited plate is evident in Figures 14-16, with Plate 2 having less energy than Plate 3, and Plate 1 having less energy than Plate 2 . The changing shape of the crossing rate curves shown in Figures 14-16 is captured both qualitatively and quantitatively by the present theory. Likewise, the probability of exceedance results shown in Figures 17-19 show a level of agreement that is consistent with the results obtained for the two plate system. This indicates that the present approach can be applied without difficulty to a multiplesubsystem built-up system . For this example, the effect of coupling on the quefrency of the response is most notable for plate 2 - this plate has relatively heavy damping compared to the other two plates. The quefrency for the uncoupled plate is 0.00656 ; for the coupled system, with forcing on Plate 3, the present theory predicts that the quefrency is 0.0181 , while the benchmark simulations yield 0.0177 . It can be noted firstly that the present theory provides an accurate estimate of the quefrency of the coupled response, and secondly that the response quefrency is significantly higher than the uncoupled value, i.e. the energy frequency response function is much more oscillatory.

One aspect of the theory that hasn't been addressed as yet is the prediction of the mean rate of peaks afforded by Eq. (25). This result requires the variance of the second derivative of the energy to be calculated using Eq. (49). The mean number of peaks over the frequency range of interest yielded by the benchmark simulations was 5.72, 5.41, and 5.63 respectively for Plates 1 to 3 . The corresponding results yielded by Eq. (25) were $7.39,7.08$, and 6.38, representing errors of $29 \%, 30 \%$, and $13 \%$. These errors reflect the approximate nature of Eq. (25), which is based on the assumption the energy rate is a Gaussian random process [11]. Nonetheless the theory correctly captures fact that the 
number of peaks greatly exceeds the number of crossings of the most crossed level, which can be read from Figures 14 to 16 as 2.47, 2.39, and 2.83, indicating that the energy is a broad banded random function of frequency.

\section{Conclusions}

This work has considered the prediction of the statistical properties of the frequency response functions of a built-up system. In common with Statistical Energy Analysis (SEA) the system has been considered to consist of an assembly of subsystems, and the concern has been with the vibrational energy of each subsystem. The existing formulations of SEA enable the mean [10] and the variance [9] of the energy to be predicted, and the present work has extended this capability to the prediction of the variance of the first and second frequency derivatives of the energy. These results can then be used to predict various properties of the energy frequency response function of each subsystem, such as crossing rates, exceedance probabilities, mean peak heights, and quefrencies. The method requires very little computation time: the standard SEA equations are solved to yield the mean energy, and the remaining equations make use of these results to compute variances and fluctuation properties. The method is based on a non-parametric GOE (Gaussian Orthogonal Ensemble) model of the system uncertainties, and this enables closed form algebraic results to be obtained in place of costly conventional Monte-Carlo simulations. The key equations are: (i) Equation (28) for the mean energy; (ii) Equation (43) for the covariance matrix of the energy; (iii) Equation (45) for the variance of the energy slope; (iv) Equation (49) for the variance of the second derivative of the energy; (v) Equation (6) for the crossing rate; (vi) Equation (22) for the quefrency; (vii) Equation (24) for the exceedance probability; (viii) Equation (25) for the mean rate of peaks; and (ix) Equation (27) for the mean trough-to-peak height.

The results have application to a range of problems of engineering interest that fall within the remit of SEA. The exceedance probability can be used to assess either the safety or the performance of an engineering system - for example a particular level of response might represent a hazard or a bound on the acceptable response. Alternatively the computed information regarding quefrencies and crossing rates could be used in response reconstruction. Traditional SEA provides only the ensemble average of the response, and fluctuations in the frequency response curve are lost in the averaging process. The present approach enables the statistical properties of these fluctuations to be computed, and this could be used as a basis for, for example, auralization.

\section{Appendix}

The analysis presented in Section 3 requires expressions for the variance of the entries of the SEA matrix $\mathbf{D}_{\text {ran }}$, together with expressions for the variance of the first and second frequency derivatives of these terms. These expressions are derived here by firstly considering a general frequency response function in the form of Eq. (3)

$$
T(\omega)=\sum_{n} a_{n} H\left(\omega, \omega_{n}\right)
$$


The mean and variance of this type of function are well established in the literature $[7,8,19,26]$, and results for the variance of the first and second frequency derivatives were derived in reference [11] for the restricted case in which the coefficients $a_{n}$ are independent of frequency. These results are adequate for the case in which Eq. (A1) represents the response of a single subsystem to prescribed external loading, but they do not cover the case in which Eq. (A1) corresponds to a matrix entry $D_{\text {ran }, j k}$ : in this case the excitation arises from a prescribed vibrational energy in subsystem $k$ and the response represents the power transmitted to subsystem $j$. For weakly coupled systems the frequency fluctuations in the coefficients $a_{n}$ will be dominated by the properties of subsystem $k$, while the frequency response function $H(\omega)$ will be that associated with subsystem $j$. In general the first and second frequency derivatives of Eq. (A1) have the form

$$
\begin{gathered}
\frac{\partial T(\omega)}{\partial \omega}=\sum_{n} a_{n} \frac{\partial H\left(\omega, \omega_{n}\right)}{\partial \omega}+\sum_{n} \frac{\partial a_{n}}{\partial \omega} H\left(\omega, \omega_{n}\right) \\
\frac{\partial^{2} T(\omega)}{\partial \omega^{2}}=\sum_{n} a_{n} \frac{\partial^{2} H\left(\omega, \omega_{n}\right)}{\partial \omega^{2}}+2 \sum_{n} \frac{\partial a_{n}}{\partial \omega} \frac{\partial H\left(\omega, \omega_{n}\right)}{\partial \omega}+\sum_{n} \frac{\partial^{2} a_{n}}{\partial \omega^{2}} H\left(\omega, \omega_{n}\right),
\end{gathered}
$$

and in order to evaluate the variance of each derivative it is first necessary to find the variance of each of the individual terms on the right hand sides of these equations. A generic term has the form

$$
X=\sum_{n} b_{n} \frac{\partial^{r} H\left(\omega, \omega_{n}\right)}{\partial \omega^{r}} \equiv \sum_{n} b_{n} H^{(r)}\left(\omega, \omega_{n}\right)
$$

and it is known from references $[19,26]$ that the variance of an expression of this type can be written as

$$
\begin{aligned}
\operatorname{Var}[X]= & \mathrm{E}\left[b_{n}^{2}\right] \int_{0}^{\infty}\left[H^{(r)}\left(\omega, \omega_{n}\right)\right]^{2} f_{1}\left(\omega_{n}\right) \mathrm{d} \omega_{n}+\operatorname{Cov}\left[b_{n}, b_{m}\right]\left\{\int_{0}^{\infty} H^{(r)}\left(\omega, \omega_{n}\right) f_{1}\left(\omega_{n}\right) \mathrm{d} \omega_{n}\right\}^{2} \ldots \\
& +\left\{\operatorname{Cov}\left[b_{n}, b_{m}\right]+\mathrm{E}\left[b_{n}\right]^{2}\right\} \int_{0}^{\infty} \int_{0}^{\infty} H^{(r)}\left(\omega, \omega_{n}\right) H^{(r)}\left(\omega, \omega_{m}\right) g_{2}\left(\omega_{n}, \omega_{m}\right) \mathrm{d} \omega_{n} \mathrm{~d} \omega_{m},
\end{aligned}
$$

where $f_{1}\left(\omega_{n}\right)=n\left(\omega_{n}\right)$ is the modal density of the receiving system, and $g_{2}\left(\omega_{n}, \omega_{m}\right)$ is known as the second correlation or cluster function (again for the receiving system). The detailed form of the second cluster function is available in the GOE literature [6], and the various integrals involved in Eq. (A5) have been evaluated in reference [11] to yield

$$
\operatorname{Var}[X]=\mathrm{E}\left[X_{0}\right]^{2} f_{1}^{2 r} \frac{\partial^{2 r}}{\partial m^{2 r}}\left\{\frac{\mathrm{E}\left[b_{n}^{2}\right]+\left(\operatorname{Cov}\left[b_{n}, b_{m}\right]+\mathrm{E}\left[b_{n}\right]^{2}\right) q(m)}{\pi m}+\operatorname{Cov}\left[b_{n}, b_{m}\right]\right\},
$$


where

$$
\mathrm{E}\left[X_{0}\right]=\sum_{n} H^{(0)}\left(\omega, \omega_{n}\right)=\int_{0}^{\infty} H^{(0)}\left(\omega, \omega_{n}\right) f_{1}\left(\omega_{n}\right) \mathrm{d} \omega_{n},
$$

and $q(m)$ is given by Eq. (15). The remaining task is to evaluate the various statistical averages of the coefficients $b_{n}$ that appear in Eq. (A6). It can be recalled that these coefficients are frequency derivatives of the coefficients $a_{n}$ that appear in Eq. (A1), and that those coefficients are in turn related to the generalised forces $g_{n}$ acting on the system. If the generalised force in mode $n$ is represented as a sum of $N$ independent complex Gaussian point loads then

$$
a_{n}=\left|g_{n}\right|^{2}, \quad g_{n}=\sum_{s=1}^{N} u_{s}(\omega) \phi_{n}\left(\mathbf{x}_{s}\right)
$$

where $u_{s}(\omega)$ is a complex Gaussian random process and $\phi_{n}\left(\mathbf{x}_{s}\right)$ is the mode shape of the receiving system at the driving point $\mathbf{x}_{s}$. If the concern is with a matrix entry $D_{\text {ran }, j k}$ then for weak coupling the statistical properties of $u_{s}(\omega)$ will be governed by the statistics of the driving subsystem $k$. If the vibration in subsystem $k$ is taken to constitute a complex diffuse wavefield, then the correlation function of $u_{s}(\omega)$ will have the form [1]

$$
R(\Omega)=\mathrm{E}\left[u_{s}^{*}(\omega) u_{s}(\omega+\Omega)\right]=\mathrm{E}\left[\left|u_{s}(\omega)\right|^{2}\right]\left(\frac{-i \eta \omega}{\Omega-i \eta \omega}\right),
$$

where $\eta$ is the effective loss factor of the subsystem. The average values of products of derivatives of the function $u_{s}(\omega)$ are then:

$$
\begin{gathered}
\mathrm{E}\left[u_{s}^{*}(\omega) u_{s}^{\prime}(\omega)\right]=\mathrm{E}\left[\left|u_{s}(\omega)\right|^{2}\right]\left(\frac{-i}{\eta \omega}\right), \quad \mathrm{E}\left[u_{s}^{\prime *}(\omega) u_{s}^{\prime}(\omega)\right]=\mathrm{E}\left[\left|u_{s}(\omega)\right|^{2}\right]\left(\frac{2}{\eta^{2} \omega^{2}}\right), \\
\mathrm{E}\left[u_{s}^{*}(\omega) u_{s}^{\prime \prime}(\omega)\right]=\mathrm{E}\left[\left|u_{s}(\omega)\right|^{2}\right]\left(\frac{-2}{\eta^{2} \omega^{2}}\right), \quad \mathrm{E}\left[u_{s}^{\prime *}(\omega) u_{s}^{\prime \prime}(\omega)\right]=\mathrm{E}\left[\left|u_{s}(\omega)\right|^{2}\right]\left(\frac{-6 i}{\eta^{3} \omega^{3}}\right), \\
\mathrm{E}\left[u_{s}^{\prime \prime *}(\omega) u_{s}^{\prime \prime}(\omega)\right]=\mathrm{E}\left[\left|u_{s}(\omega)\right|^{2}\right]\left(\frac{24}{\eta^{4} \omega^{4}}\right) .
\end{gathered}
$$

Equations (A11) -A(15) can be combined with Eqs. (A8) and (A9) to yield the following properties of the coefficients $a_{n}$ and their frequency derivatives:

$$
\mathrm{E}\left[a_{n}^{2}\right]=\mathrm{E}\left[a_{n}\right]^{2} 2(1+2 / N), \quad \operatorname{Cov}\left[a_{n}, a_{m}\right]=\mathrm{E}\left[a_{n}\right]^{2}(1 / N),
$$




$$
\begin{gathered}
\mathrm{E}\left[\frac{\partial a_{n}}{\partial \omega}\right]=0, \quad \mathrm{E}\left[\left(\frac{\partial a_{n}}{\partial \omega}\right)^{2}\right]=\mathrm{E}\left[a_{n}\right]^{2}\left\{\frac{2(1+2 / N)}{\omega^{2} \eta^{2}}\right\}, \\
\operatorname{Cov}\left[\frac{\partial a_{n}}{\partial \omega}, \frac{\partial a_{n}}{\partial \omega}\right]=\mathrm{E}\left[a_{n}\right]^{2}\left(\frac{2}{N \omega^{2} \eta^{2}}\right), \\
\mathrm{E}\left[\frac{\partial^{2} a_{n}}{\partial \omega^{2}}\right]=0, \quad \mathrm{E}\left[\left(\frac{\partial^{2} a_{n}}{\partial \omega^{2}}\right)^{2}\right]=\mathrm{E}\left[a_{n}\right]^{2}\left\{\frac{24(1+2 / N)}{\omega^{4} \eta^{4}}\right\}, \\
\operatorname{Cov}\left[\frac{\partial^{2} a_{n}}{\partial \omega^{2}}, \frac{\partial^{2} a_{m}}{\partial \omega^{2}}\right]=\mathrm{E}\left[a_{n}\right]^{2}\left(\frac{24}{N \omega^{4} \eta^{4}}\right) .
\end{gathered}
$$

These results can then be used in conjunction with Eq. (A2) to yield

$$
\operatorname{Var}\left(D_{\mathrm{ran}, j k}^{\prime}\right)=C_{j k}^{2} n_{j}^{2} \frac{\partial^{2}}{\partial m_{j}^{2}}\left[r^{2}\left(\alpha_{j k}, \gamma_{j k}, m_{j}\right)\right]+2 C_{j k}^{2}\left\{\frac{N_{k}+2+q\left(m_{j}\right)}{N_{k} \omega^{2} \eta_{k}^{2} \pi m_{j}}+\left(\frac{1}{N_{k} \omega^{2} \eta_{k}^{2}}\right)\right\}
$$

where the function $r^{2}$ is defined in Eq. (14), and

$$
\alpha_{j k}=2\left(1+2 / N_{k}\right), \quad \gamma_{j k}=1 / N_{k}
$$

Similarly, the results can be used in conjunction with Eq. (A3) to yield

$$
\begin{aligned}
\operatorname{Var}\left(D_{\text {ran }, j k}^{\prime \prime}\right)= & C_{j k}^{2} n_{j}^{4} \frac{\partial^{4}}{\partial m_{j}^{4}}\left[r^{2}\left(\alpha_{j k}, \gamma_{j k}, m_{j}\right)\right]+24 C_{j k}^{2}\left\{\frac{N_{k}+2+q\left(m_{j}\right)}{N_{k} \omega^{4} \eta_{k}^{4} \pi m_{j}}+\left(\frac{1}{N_{k} \omega^{4} \eta_{k}^{4}}\right)\right\} \\
& +8 C_{j k}^{2} n_{j}^{2} \frac{\partial^{2}}{\partial m_{j}^{2}}\left[\frac{N_{k}+2+q\left(m_{j}\right)}{N_{k} \omega^{2} \eta_{k}^{2} \pi m_{j}}\right] .
\end{aligned}
$$

The foregoing theory can be refined slightly by noting that the term $\eta \omega$ in Eq. (A10) is related to the quefrency $\sqrt{2} / \omega \eta$ of the subsystem. The use of $\eta \omega$ in the equation is equivalent to assuming that the quefrency is not affected by coupling the subsystem to other subsystems - a more accurate estimate of the quefrency for the coupled case is given by Eq. (21), which is equivalent to replacing $\eta \omega$ by $\sqrt{2} \sigma / \sigma_{s}$. Since $\sigma_{s}$ is computed on the basis of the above equations, and iterative scheme can be established whereby $\eta \omega$ is updated using the latest estimate of $\sigma_{s}$. For the numerical examples considered here, it has been found that two iterations yield a converged solution for $\sigma_{s}$ (i.e. a single repeat of the calculation with an updated estimate of the quefrency). 


\section{Funding statement}

This work was funded in part through the EPSRC Research Grant EP/P005489/1 SGRB64, Design by Science.

\section{References}

[1] Schroeder MR. Frequency-correlation functions of frequency responses in rooms. $J$. Acoust. Soc. Am. 1962; 34: 1819-1823.

[2] Lyon RH. Statistical analysis of power injection and response in structures and rooms. J. Acoust. Soc. Am. 1969; 45: 545-565.

[3] Keane AJ and Nair PB. Computational approaches for aerospace design. Hoboken: John Wiley and Sons, 2005.

[4] Soize C. A comprehensive overview of a non-parametric probabilistic approach of model uncertainties for predictive models in structural dynamics. J. Sound Vib. 2005; 288: 623-652.

[5] Weaver RL. The unreasonable effectiveness of random matrix theory for the vibrations and acoustics of complex structures. In: Wright MCM and Weaver R (eds), New directions in linear acoustics and vibration: quantum chaos, random matrix theory, and complexity. Cambridge: Cambridge University Press, 2010.

[6] Mehta ML. Random Matrices. $2^{\text {nd }}$ ed. San Diego: Academic Press, 1991.

[7] Langley RS and Brown AWM. The ensemble statistics of the energy of a random system subjected to harmonic excitation, J. Sound Vib. 2004; 275: 823-846.

[8] Weaver RL. Spectral statistics in elastodynamics, J. Acoust. Soc. Am. 1989; 85: 10051013. 
[9] Langley RS and Cotoni V. Response variance prediction in the statistical energy analysis of built-up systems, J. Acoust. Soc. Am. 2004; 115: 706-718.

[10] Lyon RH and DeJong RG. Theory and Application of Statistical Energy Analysis. Boston: Butterworth-Heinemann, 1990.

[11] Langley RS. The level crossing rates and associated statistical properties of a random frequency response function. J. Sound Vib. 2018; 417: 19-37.

[12] Randall RB. Frequency analysis. Naerum: Bruel and Kjaer, 1987.

[13] Fahy FJ. Statistical energy analysis: a critical overview. Philosophical Transactions of the Royal Society: Physical Sciences and Engineering 1994; 346: 431-447.

[14] Finnveden S. A quantitative criterion validating coupling power proportionality in statistical energy analysis. J. Sound Vib. 2011; 330: 87-109.

[15] Le Bot A and Cotoni V. Validity diagrams of statistical energy analysis. J. Sound. Vib. 2010; 329:221-235.

[16] Langley RS. The analysis of random built-up engineering systems. In: Wright MCM and Weaver R (eds), New directions in linear acoustics and vibration: quantum chaos, random matrix theory, and complexity. Cambridge: Cambridge University Press, 2010.

[17] Meirovitch L. Elements of vibration analysis. 2nd ed. New York: McGraw Hill, 1986.

[18] Kessissoglou NJ and Lucas GI. Gaussian orthogonal ensemble spacing statistics and the statistical overlap factor applied to dynamic systems. J. Sound Vib. 2009; 324: 1039-1066.

[19] Langley RS, Cicirello A and Deckers E. The ensemble statistics of the energy of a harmonically excited random system. J. Sound Vib. 2018; 413: 456-466. 
[20] Langley RS, Legault J, Woodhouse J, et al. On the applicability of the lognormal distribution in random dynamical systems. J. Sound Vib. 2013; 332: 3289-3302.

[21] Langley RS and Heron KH. Elastic wave transmission through plate/beam junctions. J. Sound. Vib. 1990; 143: 241-253.

[22] Shorter PJ and Langley RS. Vibro-acoustic analysis of complex systems. J. Sound Vib. 2005; 288: 669-699.

[23] Brody TA, Flores J, French JB, et al. Random-matrix physics: spectrum and strength fluctuations. Rev. Mod. Phys. 1981; 53: 385-479.

[24] Cotoni V, Langley RS and Kidner MRF. Numerical and experimental validation of variance prediction in the statistical energy analysis of built-up systems. J. Sound. Vib. 2005; 288: 701-728.

[25] Vanmarcke EH. On the distribution of the first-passage time for normal stationary random processes. J. Appl. Mech. 1975; 42: 215-220.

[26] Stratonovich RL, Topics in the theory of random noise. Vol. 1. New York: Gordon and Breach, 1963. 


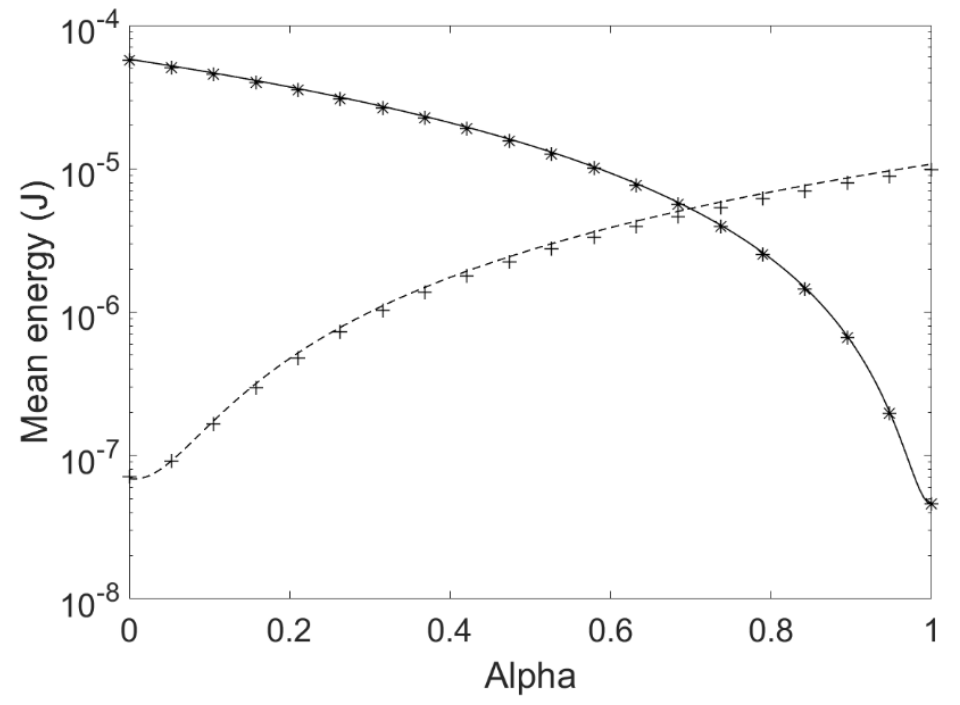

Figure 1 Mean energy as a function of the loading parameter $\alpha$. Solid curve, Plate 1 SEA prediction; dashed curve, Plate 2 SEA prediction; * benchmark results, Plate $1 ;+$ benchmark results, Plate 2.

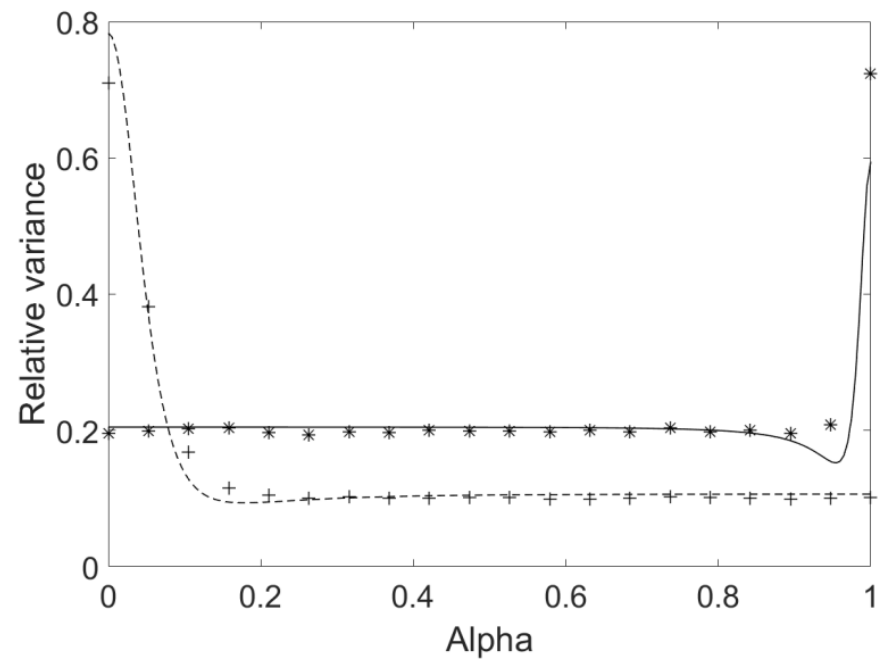

Figure 2 Relative variance of the energy as a function of the loading parameter $\alpha$. Solid curve, Plate 1 SEA prediction; dashed curve, Plate 2 SEA prediction; * benchmark results, Plate 1;+ benchmark results, Plate 2. 


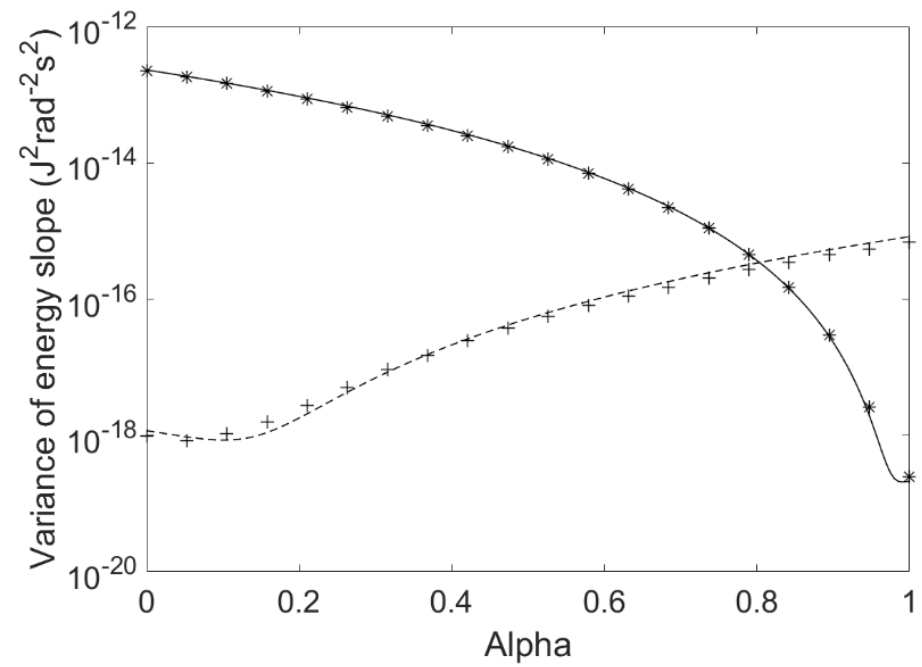

Figure 3 Variance of the energy slope as a function of the loading parameter $\alpha$. Solid curve, Plate 1 present theory; dashed curve, Plate 2 present theory; * benchmark results, Plate $1 ;+$ benchmark results, Plate 2 .

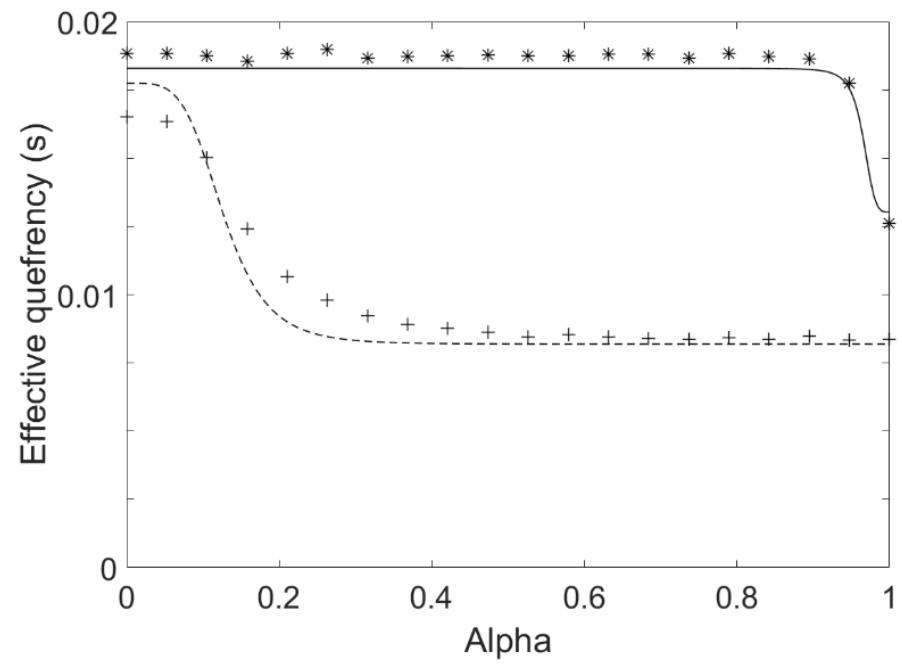

Figure 4 Effective quefrency as a function of the loading parameter $\alpha$. Solid curve, Plate 1 present theory; dashed curve, Plate 2 present theory; * benchmark results, Plate $1 ;+$ benchmark results, Plate 2 . 


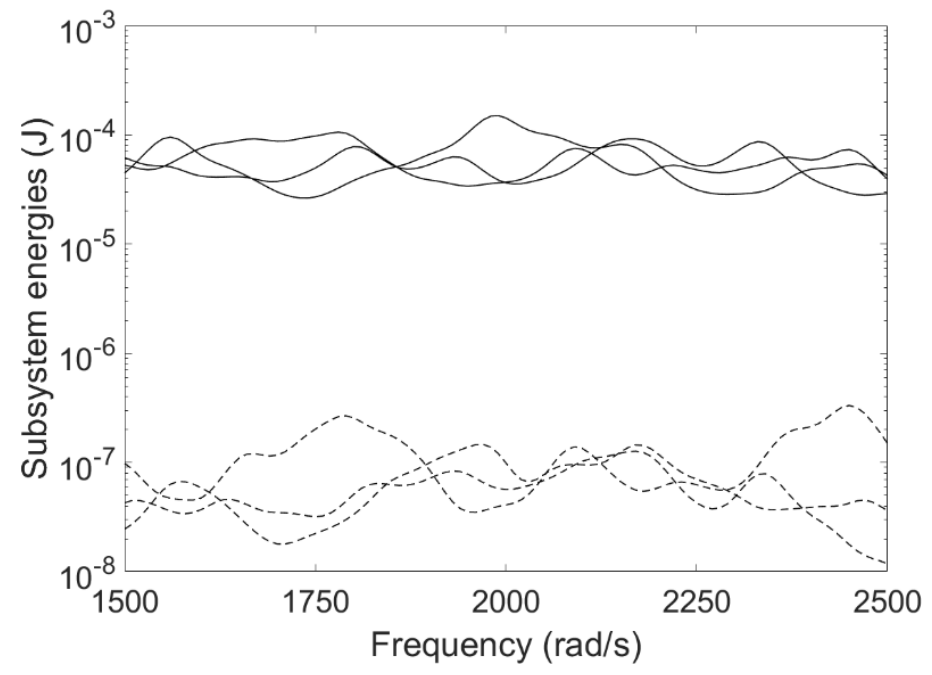

Figure 5 Three realizations of the system response when the excitation is applied to the first plate $(\alpha=0)$. Solid curves, the energy of Plate 1; dashed curves, the energy of Plate 2.

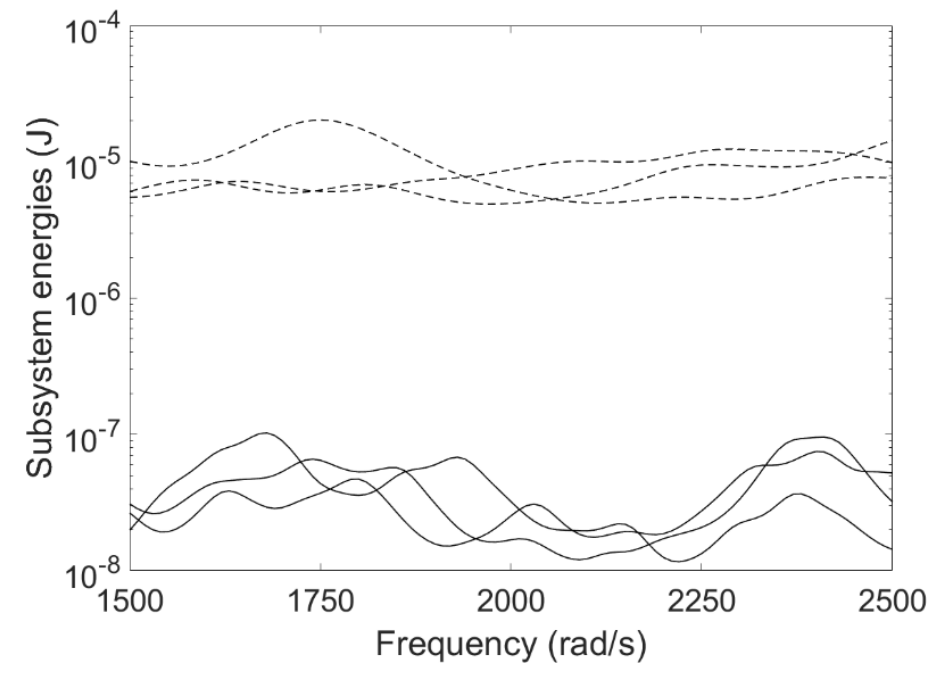

Figure 6 Three realizations of the system response when the excitation is applied to the second plate $(\alpha=1)$. Solid curves, the energy of Plate 1; dashed curves, the energy of Plate 2. 


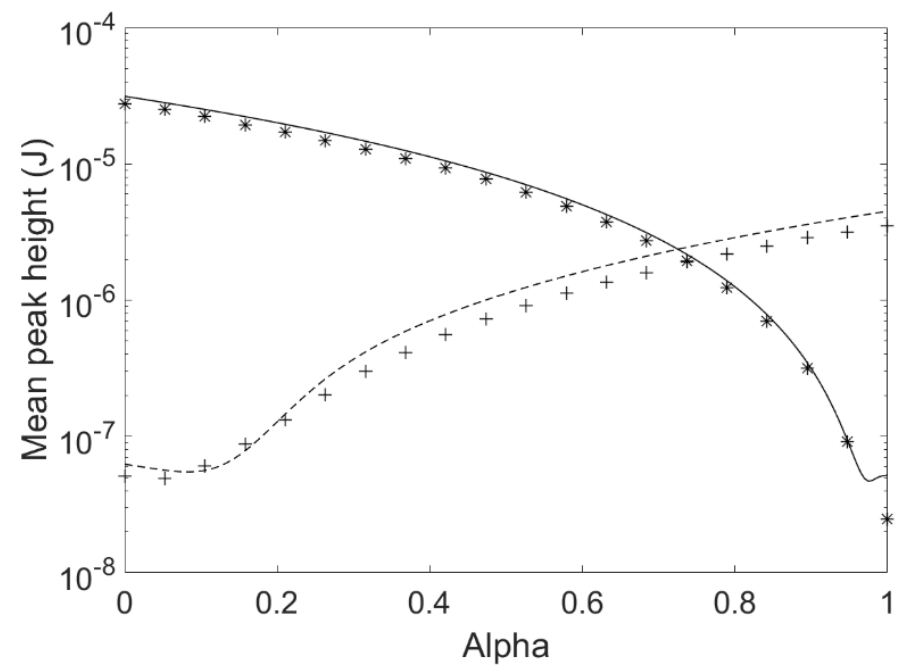

Figure 7 Mean peak height as a function of the loading parameter $\alpha$. Solid curve, Plate 1 present theory; dashed curve, Plate 2 present theory; * benchmark results, Plate 1;+ benchmark results, Plate 2.

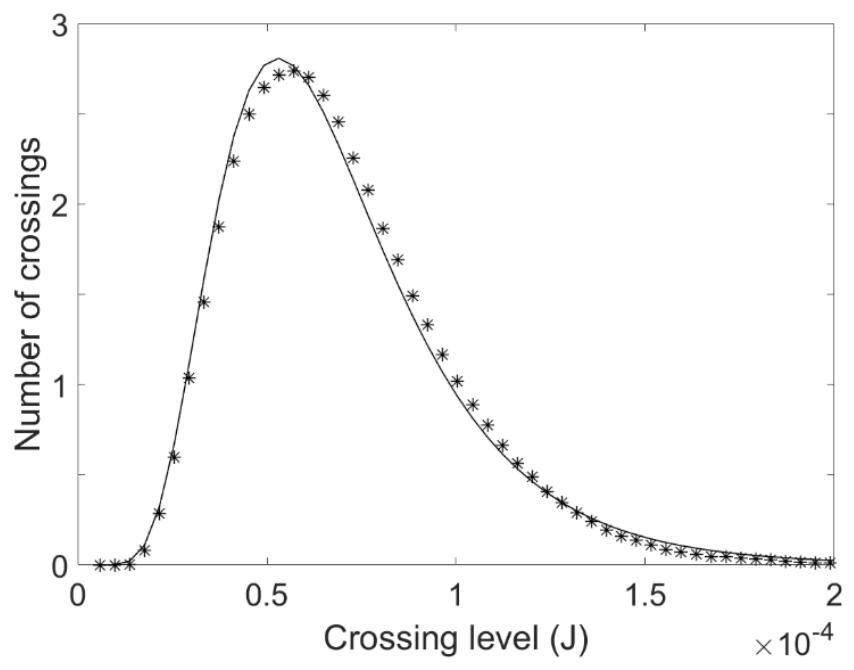

Figure 8 Mean number of crossings of the energy of Plate 1 as a function of the energy level. Solid curve, present theory; symbols, benchmark calculation. 


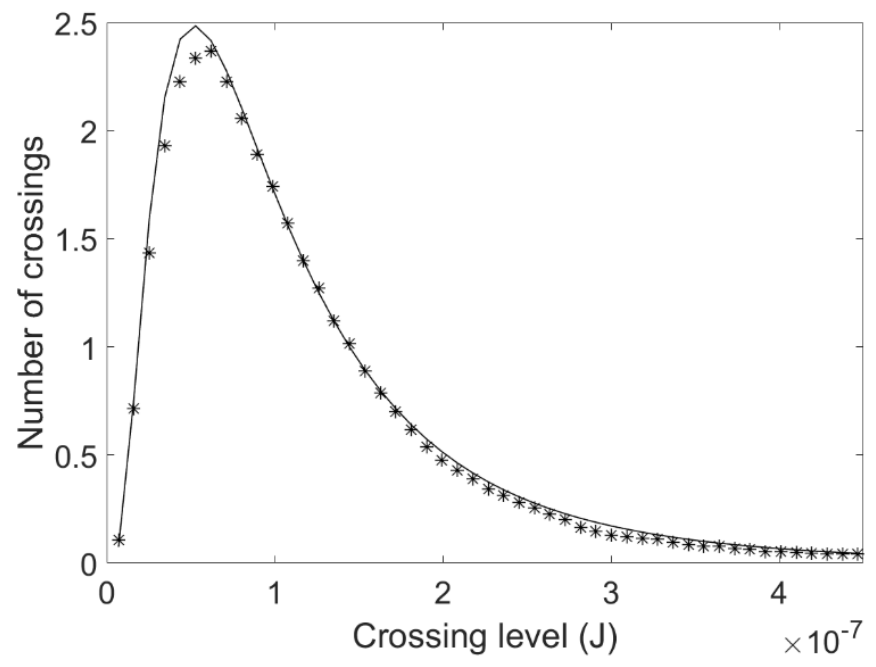

Figure 9 Mean number of crossings of the energy of Plate 2 as a function of the energy level. Solid curve, present theory; symbols, benchmark calculation.

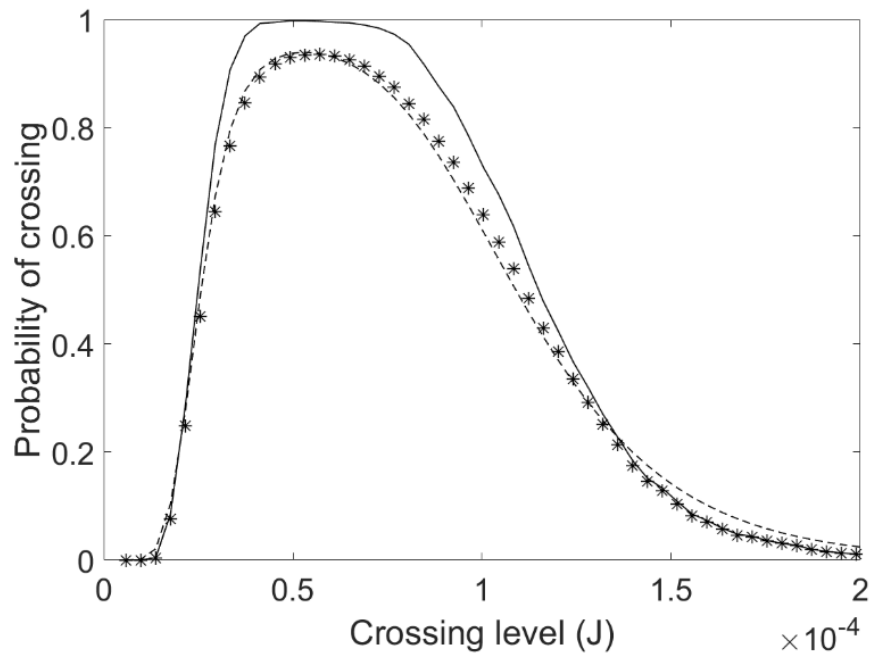

Figure 10 Probability that the energy of Plate 1 will cross a specified level at least once. Dashed curve, present theory; solid curve, benchmark calculation; symbols, result obtained when the benchmark result for the crossing rate is used in Eq. (24). 


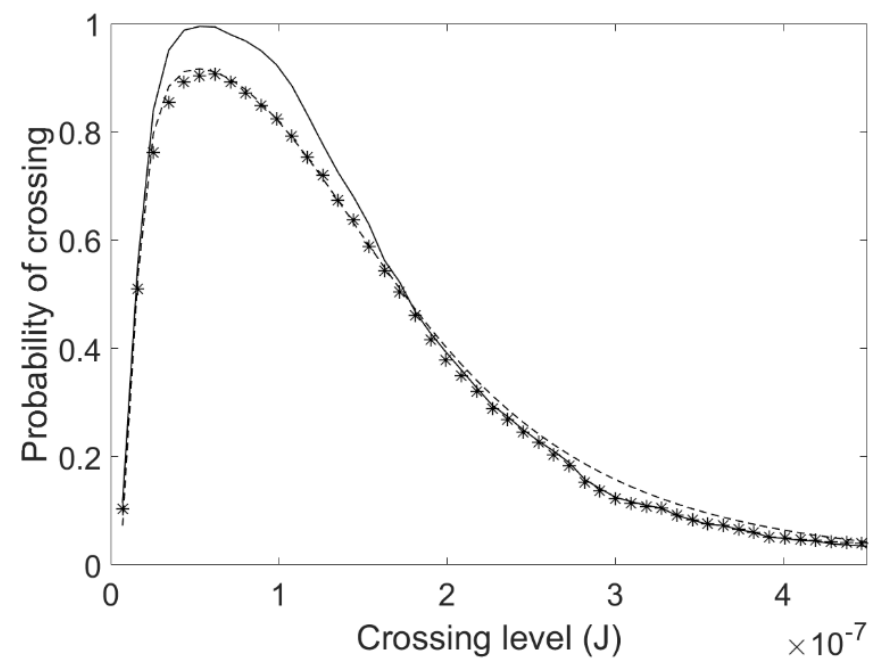

Figure 11 Probability that the energy of Plate 2 will cross a specified level at least once. Dashed curve, present theory; solid curve, benchmark calculation; symbols, result obtained when the benchmark result for the crossing rate is used in Eq. (24).

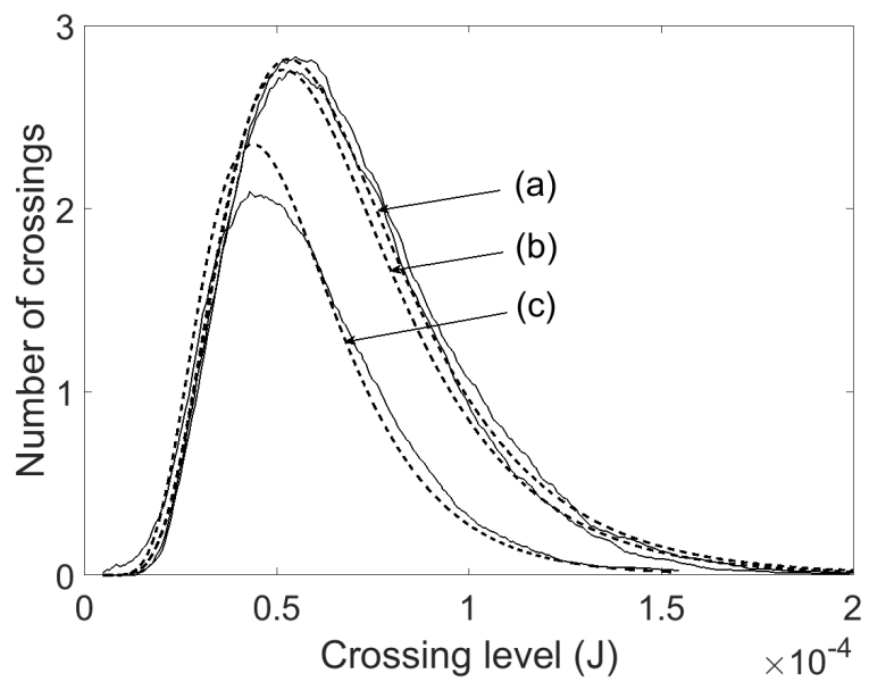

Figure 12 Probability that the energy of Plate 1 will cross a specified level at least once, for three different values of the coupling spring stiffness. Dashed curves, present theory; solid curves, benchmark calculation. (a) $k=10^{3}(\omega / 1500) \mathrm{N} / \mathrm{m}$, (b) $k=10^{4.5}(\omega / 1500) \mathrm{N} / \mathrm{m}$, (c) $k=10^{6}(\omega / 1500) \mathrm{N} / \mathrm{m}$. 


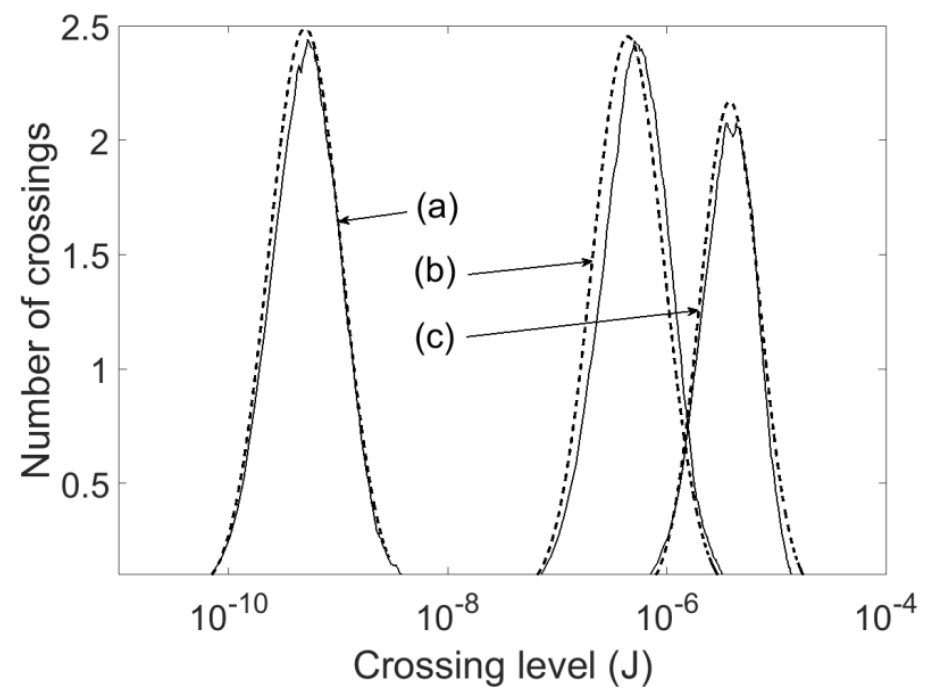

Figure 13 Probability that the energy of Plate 2 will cross a specified level at least once, for three different values of the coupling spring stiffness. Dashed curves, present theory; solid curves, benchmark calculation. (a) $k=10^{3}(\omega / 1500) \mathrm{N} / \mathrm{m}$, (b) $k=10^{4.5}(\omega / 1500) \mathrm{N} / \mathrm{m}$, (c) $k=10^{6}(\omega / 1500) \mathrm{N} / \mathrm{m}$.

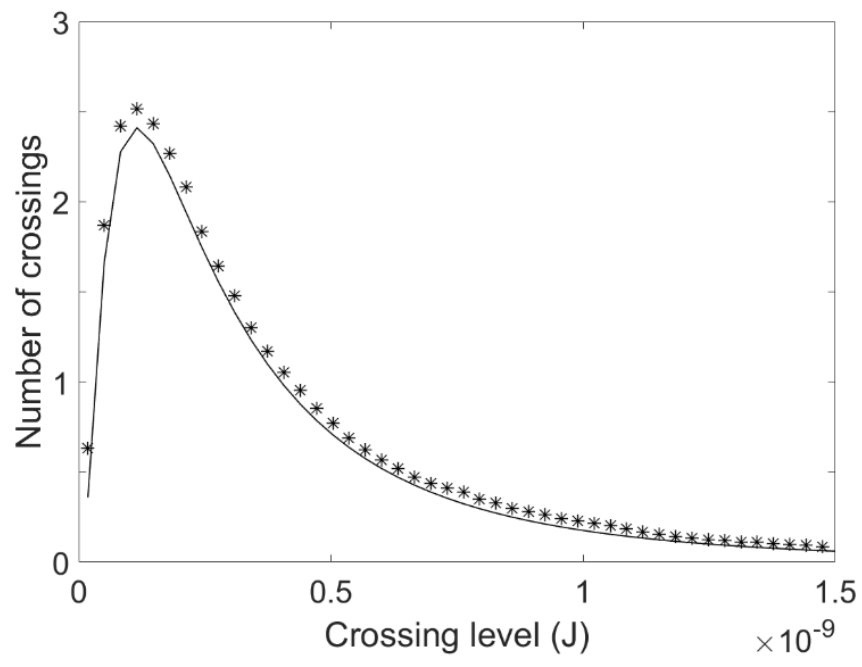

Figure 14 Mean number of crossings of the energy of Plate 1 of a three plate system as a function of the energy level. Solid curve, present theory; symbols, benchmark calculation. 


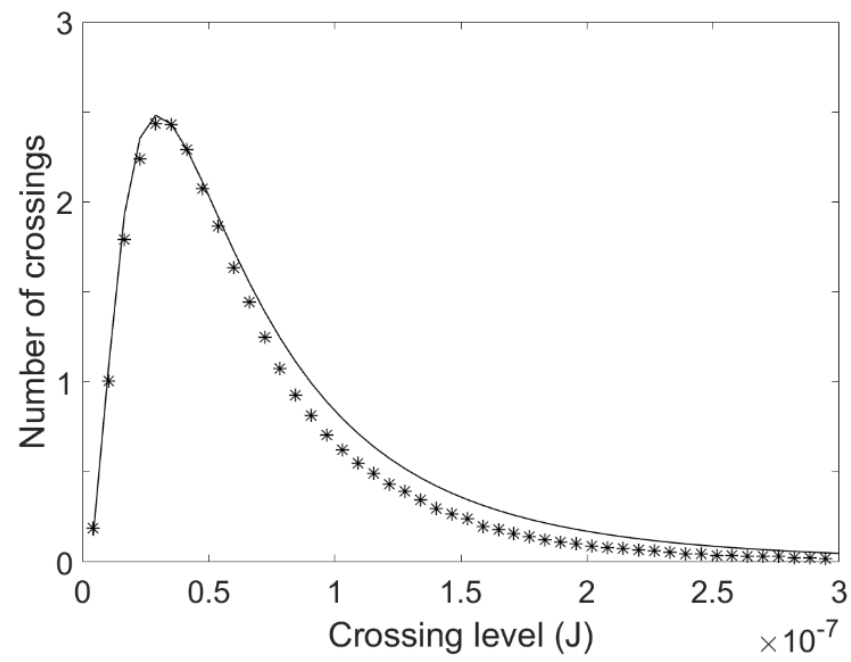

Figure 15 Mean number of crossings of the energy of Plate 2 of a three plate system as a function of the energy level. Solid curve, present theory; symbols, benchmark calculation.

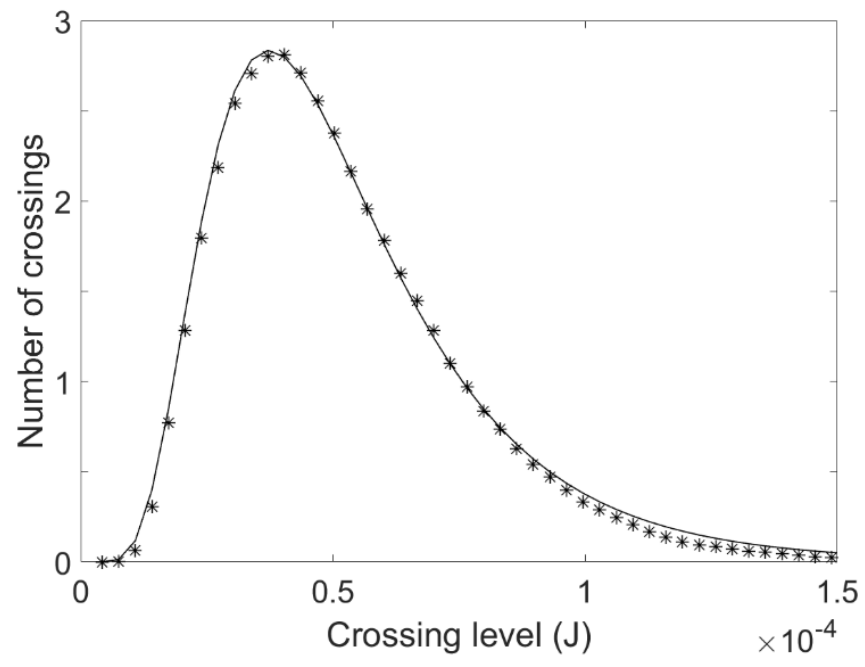

Figure 16 Mean number of crossings of the energy of Plate 3 of a three plate system as a function of the energy level. Solid curve, present theory; symbols, benchmark calculation. 


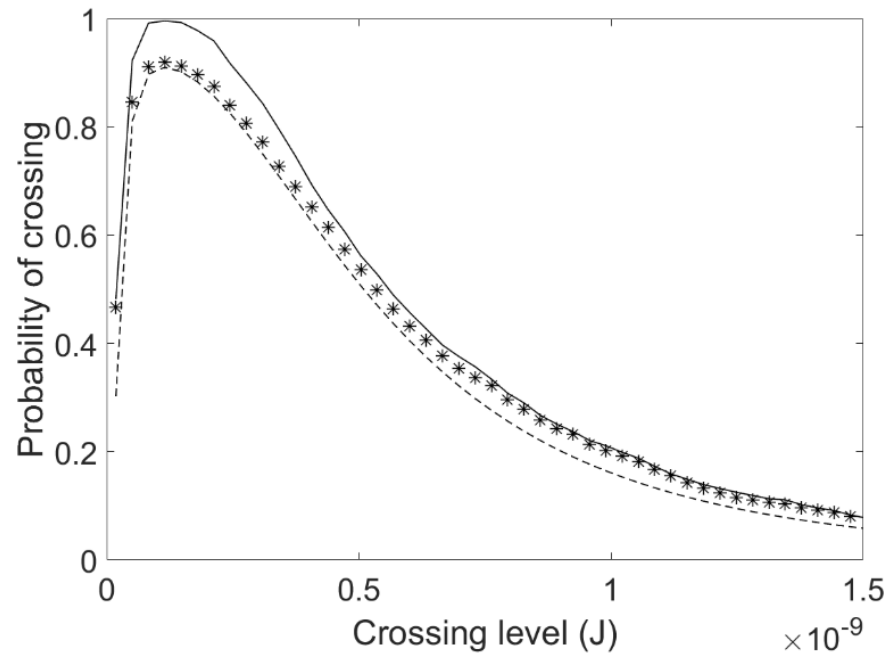

Figure 17 Probability that the energy of Plate 1 of a three plate system will cross a specified level at least once. Dashed curve, present theory; solid curve, benchmark calculation; symbols, result obtained when the benchmark result for the crossing rate is used in Eq. (24).

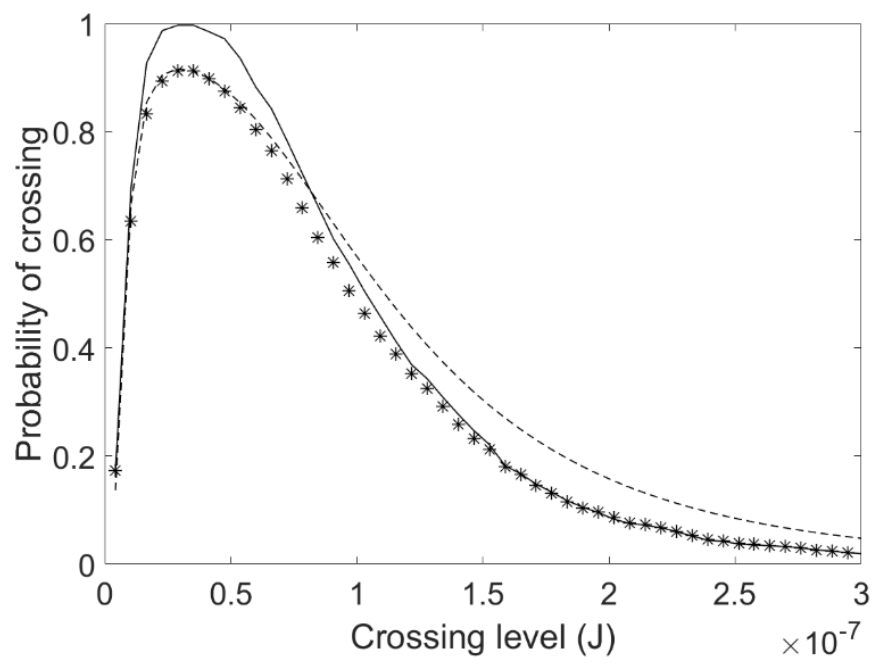

Figure 18 Probability that the energy of Plate 2 of a three plate system will cross a specified level at least once. Dashed curve, present theory; solid curve, benchmark calculation; symbols, result obtained when the benchmark result for the crossing rate is used in Eq. (24). 


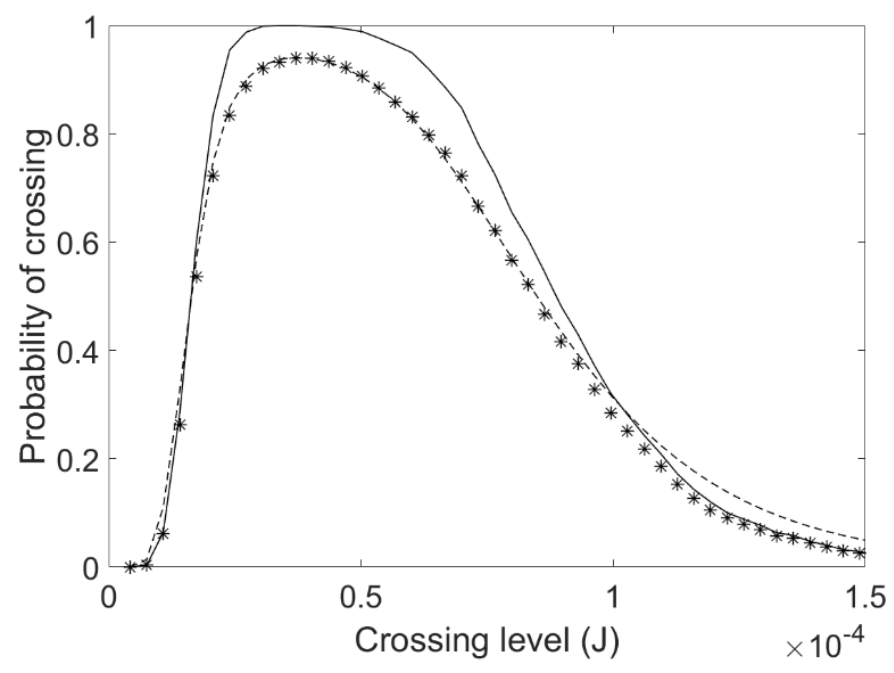

Figure 19 Probability that the energy of Plate 3 of a three plate system will cross a specified level at least once. Dashed curve, present theory; solid curve, benchmark calculation; symbols, result obtained when the benchmark result for the crossing rate is used in Eq. (24). 